\title{
A COMPLETE CLASSIFICATION OF BIFURCATION DIAGRAMS OF A $P$-LAPLACIAN DIRICHLET PROBLEM II. GENERALIZED NONLINEARITIES
}

\author{
Feng-Lin Wang and Shin-Hwa Wang*
}

Abstract. We study the bifurcation diagrams of classical positive solutions $u$ with $\|u\|_{\infty} \in(0, \infty)$ of the $p$-Laplacian Dirichlet problem

$$
\left\{\begin{array}{l}
\left(\varphi_{p}\left(u^{\prime}(x)\right)\right)^{\prime}+\lambda f_{q, r}(u(x))=0,-1<x<1, \\
u(-1)=0=u(1)
\end{array}\right.
$$

where $p>1, \varphi_{p}(y)=|y|^{p-2} y,\left(\varphi_{p}\left(u^{\prime}\right)\right)^{\prime}$ is the one-dimensional $p$-Laplacian, $\lambda>0$ is a bifurcation parameter, and

$$
f_{q, r}(u)= \begin{cases}|1-u|^{q}, & \text { if } 0<u \leq 1, \\ |1-u|^{r}, & \text { if } u>1,\end{cases}
$$

with positive constants $q$ and $r$. We give explicit formulas of bifurcation curves of classical positive solutions on the $\left(\lambda,\|u\|_{\infty}\right)$-plane. More importantly, for different $(p, q, r)$, we give a complete classification of all bifurcation diagrams. Hence we are able to determine the (exact) multiplicity of classical positive solutions for each $(p, q, r, \lambda)$. Our results generalize the results of Lee et al. [J. Math. Anal. Appl., 330 (2007), 276-290] with nonlinearity $f_{q, r}$ generalized from $q=r>0$ to $q, r>0$.

\section{INTRODUCTION}

This paper is a continuation of the paper by Lee et al. [4]. In the present paper, we study the bifurcation diagrams of classical positive solutions $u$ with $\|u\|_{\infty} \in(0, \infty)$ of the $p$-Laplacian Dirichlet problem

$$
\left\{\begin{array}{l}
\left(\varphi_{p}\left(u^{\prime}(x)\right)\right)^{\prime}+\lambda f_{q, r}(u(x))=0, \quad-1<x<1, \\
u(-1)=0=u(1)
\end{array}\right.
$$

Received January 24, 2011, accepted August 20, 2011.

Communicated by Eiji Yanagida.

2010 Mathematics Subject Classification: 34B18, 35B32.

Key words and phrases: Bifurcation diagram, Positive solution, Exact multiplicity, $p$-Laplacian, Time map.

This was supported partially supported by the National Science Council, Republic of China.

*Corresponding author. 
where $p>1, \varphi_{p}(y)=|y|^{p-2} y,\left(\varphi_{p}\left(u^{\prime}\right)\right)^{\prime}$ is the one-dimensional $p$-Laplacian, $\lambda>0$ is a bifurcation parameter, and the nonlinearity

$$
f_{q, r}(u)= \begin{cases}|1-u|^{q}, & \text { if } 0<u \leq 1, \\ |1-u|^{r}, & \text { if } u>1,\end{cases}
$$

with positive constants $q$ and $r$. Notice that the nonlinearity $f_{q, r}(u)$ in the $p$-Laplacian problem (1.1) satisfies hypotheses (H1)-(H3):

(H1) $f_{q, r}(u) \in C[0, \infty) \cap C^{\infty}([0,1) \cup(1, \infty))$,

(H2) $f_{q, r}(1)=0, f_{q, r}(u)>0$ for $u \geq 0, u \neq 1$,

(H3) $f_{q, r}(u)$ satisfies a locally Lipschitz condition of order $p-1$ at $u=1^{-}$if and only if $q \geq p-1$, and $f_{q, r}(u)$ satisfies a locally Lipschitz condition of order $p-1$ at $u=1^{+}$if and only if $r \geq p-1$.

When $p=2$, among other results, Smoller and Wasserman [9, Section 2, pp. 276-277] studied the exact multiplicity of positive solutions of the Laplacian Dirichlet problem

$$
\left\{\begin{array}{l}
u^{\prime \prime}(x)+\lambda f(u(x))=0, \quad-1<x<1 \\
u(-1)=0=u(1)
\end{array}\right.
$$

where the nonlinearity

$$
f=f^{*}(u)=(u-1)^{2}(u-c), 0<1<c<\infty,
$$

satisfies $\left(\mathrm{H}^{\prime}\right) f^{*}(u) \in C^{\infty}[0, \infty)$ and $\left(\mathrm{H} 2^{\prime}\right) f^{*}(1)=f^{*}(c)=0, f^{*}(u)>0$ for $u \in(0,1) \cup(1, c)$, and $f^{*}(u)<0$ for $u \in(c, \infty)$. (Cf. [7, Section 5] for numerical simulations of (1.3) for similar general nonlinearities $f$.) Similarly, when $p=2$ and assuming that a general nonlinearity $f$ satisfies $\left(\mathrm{H} 2^{\prime}\right)$ with $f^{*}$ replaced by $f$, and that $f$ is a locally Lipschitz continuous function on $(0, \infty)$, P. L. Lions [6, Section 3.2] studied the existence and multiplicity of positive solutions of

$$
\begin{cases}\Delta u+\lambda f(u)=0 & \text { in } \Omega, \\ u=0 & \text { on } \partial \Omega,\end{cases}
$$

where $\Omega$ is a bounded regular domain in $\mathbb{R}^{N}, N \geq 1$.

For (1.1) with $q=r>0$ and

$$
f_{q, r}(u)=f_{q, q}(u)=|1-u|^{q} \quad \text { for } 0 \leq u<\infty,
$$

Lee et al. [4] studied (classified) bifurcation diagrams of (classical) positive solutions of (1.1) and they gave the exact multiplicity of classical positive solutions for each $(p, q, r, \lambda)$; see [4, Theorem 2.1 and Corollary 2.2] for details. 
In this paper, we generalize [4, Theorem 2.1 and Corollary 2.2] to (1.1) for more general nonlinearity $f_{q, r}$ in (1.2) with $q, r>0$. We study (classified) bifurcation diagrams of (classical) positive solutions of (1.1) by modify the time-map method (quadrature method) used in [4]. The next Proposition 1.1 on some properties of the corresponding time map formula $T_{f_{q, r}}(\alpha)$ of (1.1) slightly generalized [4, Proposition 1.1]. Proposition 1.1 mainly follows by applying similar arguments in the proofs of [3, Theorems 2.1, 2.5-2.7 and 2.9-2.10] and by [5, Theorem 5.3]; we omit the proofs. (Note that, for Proposition 1.1(i), it is well-known and easy to prove that $T_{f_{q, r}}(\alpha)$ is strictly increasing on $(0,1)$ since $(p-1) f_{q, r}(u)-u f_{q, r}^{\prime}(u)=(1-u)^{q-1}[(p-1)(1-u)+q u]>0$ on $(0,1)$ for $p>1, q>0$.)

Define

$$
F_{q, r}(u)=\int_{0}^{u} f_{q, r}(t) d t= \begin{cases}\frac{1}{q+1}\left[1-(1-u)^{q+1}\right], & \text { if } 0<u \leq 1, \\ \frac{1}{q+1}\left[\frac{q+1}{r+1}(u-1)^{r+1}+1\right], & \text { if } u>1\end{cases}
$$

and

$$
I_{f_{q, r}}= \begin{cases}(0, \infty), & \text { if } \lim _{\alpha \rightarrow 1^{-}} \int_{0}^{\alpha} \frac{d u}{\left[F_{q, r}(\alpha)-F_{q, r}(u)\right]^{1 / p}}<\infty \\ (0,1) \cup(1, \infty), & \text { otherwise. }\end{cases}
$$

Proposition 1.1. Consider (1.1) with constants $p>1$ and $q, r>0$. Then, given $\lambda>0$, there exists a unique (classical) positive solution to (1.1) with $\|u\|_{\infty}=u(0)=\alpha$ if and only if

$$
T_{f_{q, r}}(\alpha) \equiv\left(\frac{p-1}{p}\right)^{1 / p} \int_{0}^{\alpha} \frac{d u}{\left[F_{q, r}(\alpha)-F_{q, r}(u)\right]^{1 / p}}=\lambda^{1 / p} \text { for } \alpha \in I_{f_{q, r}} .
$$

Moreover, $T_{f_{q, r}}(\alpha)$ satisfies

(i) $T_{f_{q, r}}(\alpha) \in C((0,1) \cup(1, \infty))$ and $T_{f_{q, r}}(\alpha)$ is strictly increasing on $(0,1)$.

(ii) $\lim _{\alpha \rightarrow 0^{+}} T_{f_{q, r}}(\alpha)=0$.

(iii)

(iv)

$$
\lim _{\alpha \rightarrow 1^{-}} T_{f_{q, r}}(\alpha)= \begin{cases}{\left[\frac{(p-1)(q+1)}{p}\right]^{1 / p} \frac{p}{p-1-q},} & \text { if } q<p-1 \\ \infty, & \text { if } q \geq p-1\end{cases}
$$

$$
\lim _{\alpha \rightarrow \infty} T_{f_{q, r}}(\alpha)= \begin{cases}\infty, & \text { if } r<p-1 \\ (p-1)^{1 / p} \frac{\pi}{p} \csc \frac{\pi}{p}, & \text { if } r=p-1 \\ 0, & \text { if } r>p-1\end{cases}
$$




\section{Main Results}

The main results in this paper are next Theorems 2.1 and 2.2, and Corollary 2.3. First, in Theorem 2.1, we give explicit formulas of the time map $T_{f_{q, r}}(\alpha)\left(=\lambda^{1 / p}\right)$ of (1.1). Subsequently, in Theorem 2.2, for different $(p, q, r)$, we give a classification of bifurcation diagrams of (classical) positive solutions of (1.1) on the $\left(\|u\|_{\infty}, \lambda^{1 / p}\right)$ plane. Hence, in Corollary 2.3, we are able to determine the (exact) multiplicity of (classical) positive solutions of (1.1) for each $(p, q, r, \lambda)$. We note that Theorem 2.2 generalizes [4, Theorem 2.1], and Corollary 2.3 generalizes [4, Corollary 2.2]. We also note that Theorem 2.2 and Fig. 1 suggest all possible bifurcation diagrams of (classical) positive solutions of $p$-Laplacian problem (1.1) with $f_{q, r}(u)$ replaced by general nonlinearities $f$ satisfying the following hypotheses:

$\left(\mathrm{H} 1^{\prime \prime}\right) f(u) \in C[0, \infty) \cap C^{2}([0,1) \cup(1, \infty))$,

$\left(\mathrm{H} 2^{\prime \prime}\right) f(1)=0, f(u)>0$ for $u \geq 0, u \neq 1$, and $\lim _{u \rightarrow \infty} f(u)=\infty$,

$\left(\mathrm{H}^{\prime}\right)$ The convexity of $f(u)$ keeps one sign on $(0,1)$ and on $(1, \infty)$, respectively.

Further studies on this problem are necessary.

We first recall some special functions and Euler's constant used in the main results as follows (see e.g. [10, pp. 44-45 and 56] and [8, pp. 34 and 184 ]):

(i) the gamma function

$$
\Gamma(x)=\int_{0}^{\infty} t^{x-1} e^{-t} d t \quad(x \in \mathbb{R} \backslash\{0,-1,-2, \cdots\}),
$$

(ii) the psi function

$$
\psi(x)=\frac{d}{d x} \ln \Gamma(x), \quad(x \in \mathbb{R} \backslash\{0,-1,-2, \cdots\}),
$$

(iii) the hypergeometric function

$$
F(a, b ; c ; x)=\sum_{n=0}^{\infty} \frac{(a)_{n}(b)_{n}}{n !(c)_{n}} x^{n},
$$

where $|x|<1, a, b, c \in \mathbb{R}, c \neq 0,-1,-2, \ldots$ and Pochhammer's symbol

$$
(a)_{n} \equiv \begin{cases}1, & \text { if } n=0, \\ a(a+1) \cdots(a+n-1), & \text { if } n \geq 1,\end{cases}
$$

(iv) the generalized hypergeometric function

$$
{ }_{P} F_{Q}\left(a_{1}, a_{2}, \cdots, a_{P} ; b_{1}, b_{2}, \cdots, b_{Q} ; x\right)=\sum_{n=0}^{\infty} \frac{\left(a_{1}\right)_{n} \cdots\left(a_{P}\right)_{n}}{n !\left(b_{1}\right)_{n} \cdots\left(b_{Q}\right)_{n}} x^{n},
$$

where $P$ is the number of the numerator parameters $a_{i} \in \mathbb{R}, Q$ is the number of the denominator parameters $b_{j} \in \mathbb{R}, b_{j} \neq 0,-1,-2, \ldots$, and $|x|<1$, 
(v) Euler's constant

$$
\gamma \equiv \lim _{n \rightarrow \infty}\left(\sum_{m=1}^{n} \frac{1}{m}-\ln n\right) \approx 0.5772 .
$$

Theorem 2.1. Consider (1.1) with constants $p>1$ and $q, r>0$. Then the following assertions $(A)$ and $(B)$ hold:

(A) For $0<\alpha<1$,

(I) If $q \neq p-1$, then

$$
\begin{aligned}
T_{f_{q, r}}(\alpha)= & {\left[\frac{(p-1)(q+1)}{p}\right]^{1 / p}\left[\frac{\Gamma\left(\frac{p-1}{p}\right) \Gamma\left(\frac{q+1-p}{p q+p}\right)}{(q+1) \Gamma\left(\frac{q}{q+1}\right)}(1-\alpha)^{(p-1-q) / p}\right.} \\
& \left.-\frac{p}{q+1-p} F\left(\frac{1}{p}, \frac{q+1-p}{p q+p} ; \frac{p q+q+1}{p q+p} ;(1-\alpha)^{q+1}\right)\right] .
\end{aligned}
$$

(II) If $q=p-1$, then

(B) For $\alpha>1$,

$$
\begin{aligned}
T_{f_{q, r}}(\alpha)= & \frac{(p-1)^{1 / p}}{p^{2}}\left\{p\left[p \ln \left(\frac{1}{1-\alpha}\right)-\gamma-\psi\left(\frac{p-1}{p}\right)\right]\right. \\
& \left.-(1-\alpha)^{p}{ }_{3} F_{2}\left(1,1, \frac{p+1}{p} ; 2,2 ;(1-\alpha)^{p}\right)\right\}
\end{aligned}
$$

$$
\begin{aligned}
& T_{f_{q, r}}(\alpha) \\
= & {\left[\frac{(p-1)(r+1)}{p}\right]^{1 / p}(\alpha-1)^{(p-1-r) / p} } \\
& \times\left[\frac{1}{\alpha-1} F\left(\frac{1}{p}, \frac{1}{q+1} ; \frac{q+2}{q+1} ;-\frac{r+1}{q+1}(\alpha-1)^{-(r+1)}\right)+\frac{\Gamma\left(\frac{r+2}{r+1}\right) \Gamma\left(\frac{p-1}{p}\right)}{\Gamma\left(\frac{p r+2 p-r-1}{p r+p}\right)}\right] .
\end{aligned}
$$

Remark 1. In (2.4), for $p>1$, we have

$$
\gamma+\psi\left(\frac{p-1}{p}\right)=-\sum_{j=0}^{\infty}\left(\frac{1}{\frac{p-1}{p}+j}-\frac{1}{j+1}\right)<0
$$

by applying Lemma 3.1(xv) stated below.

Theorem 2.2. ([See Figs. 1 and 2]). Consider (1.1) with constants $p>1$ and $q, r>0$. Then the following assertions $(A)$ and $(B)$ hold: 


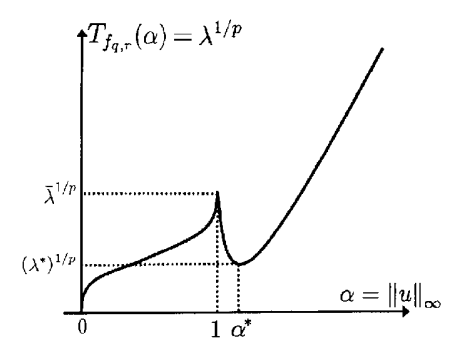

(a)

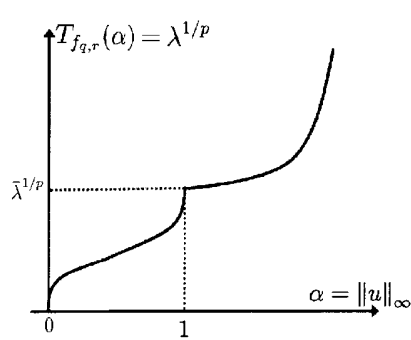

(b)

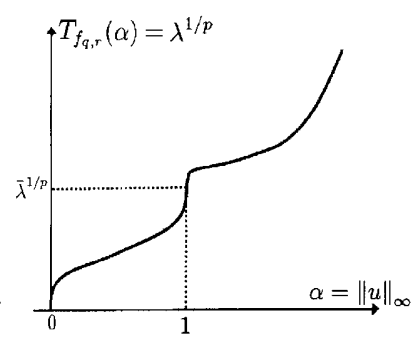

(c1)

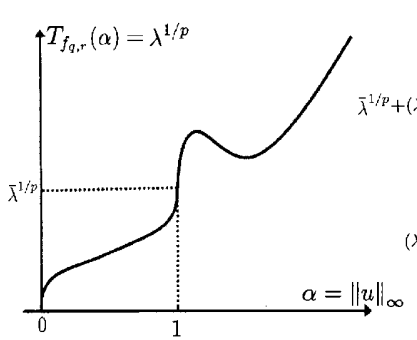

(c2)

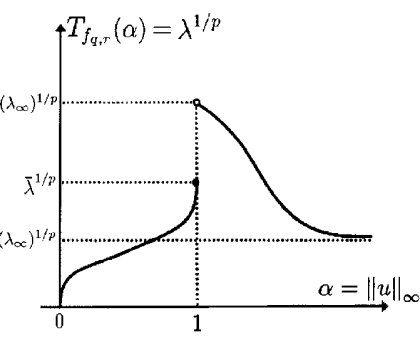

(d)

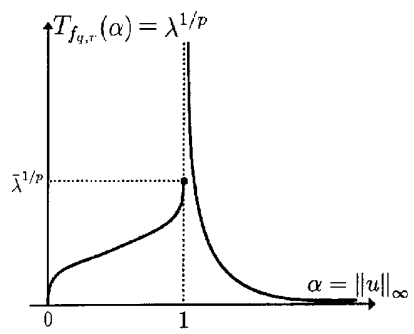

(e)

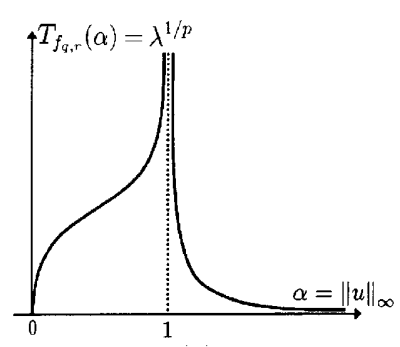

(h)

Fig. 1. Classified bifurcation diagrams $T_{f_{q, r}}(\alpha)$ of (1.1). $\bar{\lambda}=\frac{(p-1)(q+1)}{p}\left(\frac{p}{p-1-q}\right)^{p}$ and $\lambda_{\infty}=(p-1)\left(\frac{\pi}{p} \csc \frac{\pi}{p}\right)^{p}$. (a) $(\max \{0,(p-2) / 2\}<q=r<p-1)$ or $(r<q<$ $p-1)$. (b) $q=r=(p-2) / 2>0$. (c1) $(q=r<(p-2) / 2$ and $p>2)$ or (for some $q, r$ with $q<r<p-1$ ). (c2) (for some $q, r$ with $q<r<p-1$ ). (d) $q<r=p-1$. (e) $q<p-1<r$. (f) $q \geq p-1>r$. (g) $q \geq r=p-1$. (h) $q \geq p-1$ and $r>p-1$.

(A) If $q<p-1$, then

(I) If $r<p-1$, then

(i) $\lim _{\alpha \rightarrow 0^{+}} T_{f_{q, r}}(\alpha)=0, \lim _{\alpha \rightarrow \infty} T_{f_{q, r}}(\alpha)=\infty$, and

$$
\begin{aligned}
\lim _{\alpha \rightarrow 1^{-}} T_{f_{q, r}}(\alpha) & =\left[\frac{(p-1)(q+1)}{p}\right]^{1 / p} \frac{p}{p-1-q} \\
\equiv(\bar{\lambda})^{1 / p} \equiv T_{f_{q, r}}(1) & =\lim _{\alpha \rightarrow 1^{+}} T_{f_{q, r}}(\alpha) .
\end{aligned}
$$

(ii) $T_{f_{q, r}}(\alpha) \in C(0, \infty)$ and $T_{f_{q, r}}(\alpha)$ is strictly increasing on $(0,1]$. 


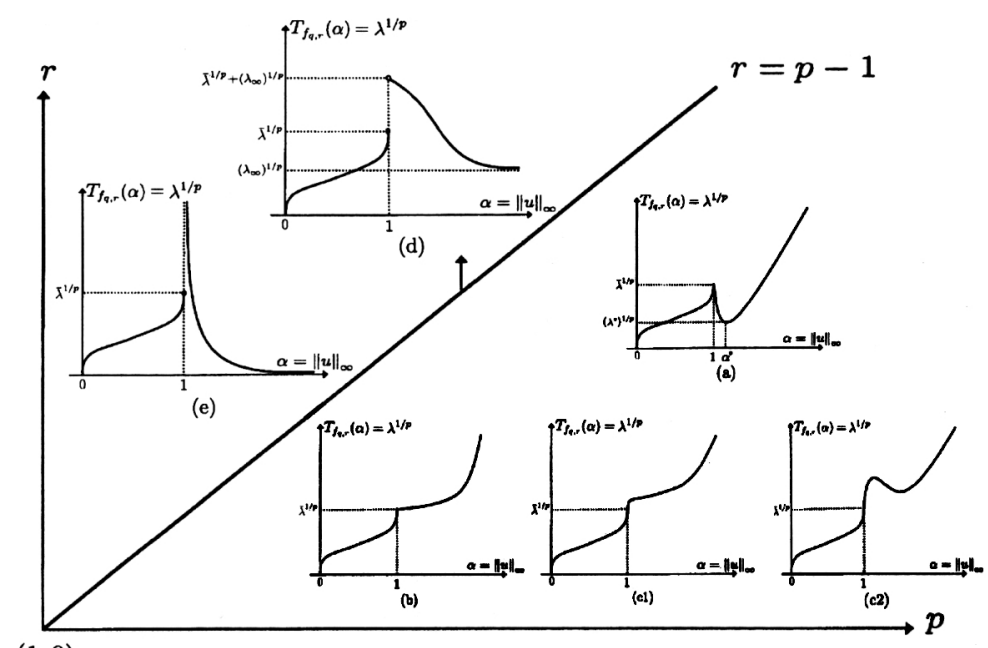

(A) $q<p-1$

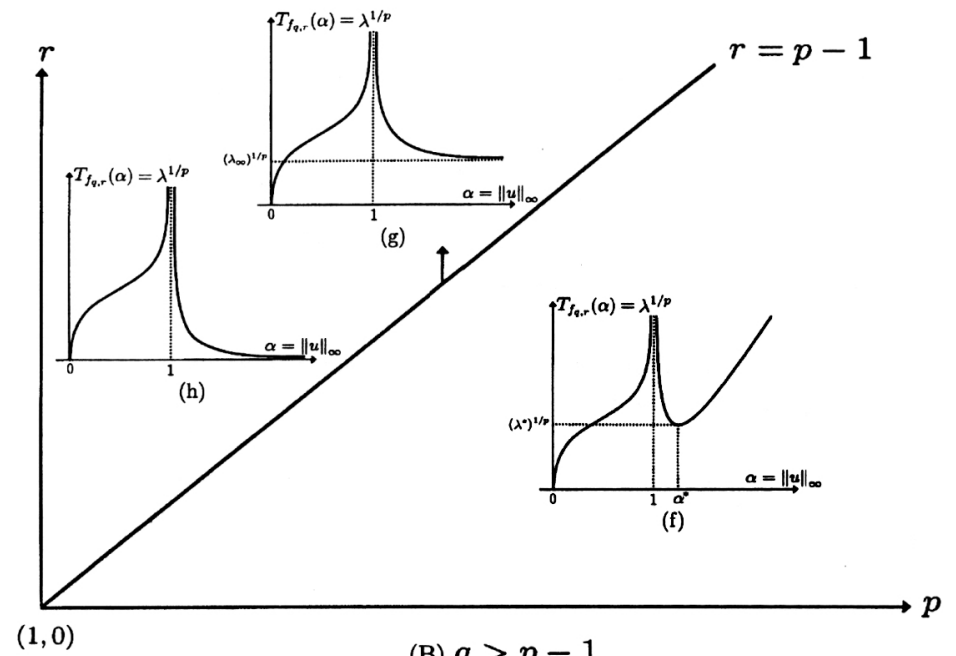

(B) $q \geq p-1$

Fig. 2. Classified bifurcation diagrams $T_{f_{q, r}}(\alpha)$ of (1.1), drawn on the $(p, r)$-plane with $p>1$ and $q, r>0$. (A) $q<p-1$. (B) $q \geq p-1$.

(iii)

$$
\begin{aligned}
& \lim _{\alpha \rightarrow 1^{+}} T_{f_{q, r}}^{\prime}(\alpha) \\
& = \begin{cases}-\infty, & \text { if }(\max \{0,(p-2) / 2\}<q=r<p-1) \\
& \text { or }(r<q<p-1),(\text { see Fig. } 1(a)) \\
0, & \text { if } q=r=(p-2) / 2>0,(\text { see Fig. } 1(b)) \\
\infty, & \text { if }(q=r<(p-2) / 2 \text { and } p>2) \text { or }(q<r<p-1) \\
& \text { (see Fig. } 1(c 1) \text { and }(c 2)) .\end{cases}
\end{aligned}
$$


(iv) If $(\max \{0,(p-2) / 2\}<q=r<p-1)$ or $(r<q<p-1)$, then $T_{f_{q, r}}(\alpha)$ has at least one critical point at some $\alpha^{*}$, a local minimum, on $(1, \infty)$. In particular, if $\max \{0,(p-2) / 2\}<q=r<p-1$, then $T_{f_{q, q}}(\alpha)$ has exactly one critical point at some $\alpha^{*}$, a local minimum, on $(1, \infty)$, and for fixed $p>1$, the number $\alpha^{*}=\alpha^{*}(q)$ satisfies

$$
\lim _{q \rightarrow(p-1)^{-}} \alpha^{*}(q)=\infty \text { and } \lim _{q \rightarrow(\max \{0,(p-2) / 2\})^{+}} \alpha^{*}(q)=1 .
$$

(Note that numerical simulations suggest that, for fixed $p>1, \alpha^{*}(q)$ is a strictly increasing function of $q \in(\max \{0,(p-2) / 2\}, p-1)$. But we are not able to give a proof.)

(v) If $q=r \leq(p-2) / 2$ and $p>2$, then $T_{f_{q, r}}(\alpha)$ is a strictly increasing function on $[1, \infty)$.

(See Fig. 1(c1) and (c2), and see Remark 2 stated below for $T_{f_{q, r}}(\alpha)$ in the case $q<r<p-1$.)

(II) If $r=p-1$, then

(i) $\lim _{\alpha \rightarrow 0^{+}} T_{f_{q, r}}(\alpha)=0$,

$$
\begin{aligned}
& \lim _{\alpha \rightarrow \infty} T_{f_{q, r}}(\alpha)=(p-1)^{1 / p} \frac{\pi}{p} \csc \frac{\pi}{p} \equiv\left(\lambda_{\infty}\right)^{1 / p}, \\
& \lim _{\alpha \rightarrow 1^{-}} T_{f_{q, r}}(\alpha)=\left[\frac{(p-1)(q+1)}{p}\right]^{1 / p} \frac{p}{p-1-q} \equiv(\bar{\lambda})^{1 / p} \equiv T_{f_{q, r}}(1), \\
& \text { and } \\
& \lim _{\alpha \rightarrow 1^{+}} T_{f_{q, r}}(\alpha)=\left[\frac{(p-1)(q+1)}{p}\right]^{1 / p} \frac{p}{p-1-q}+(p-1)^{1 / p} \frac{\pi}{p} \csc \frac{\pi}{p} \\
& =\lim _{\alpha \rightarrow 1^{-}} T_{f_{q, r}}(\alpha)+\lim _{\alpha \rightarrow \infty} T_{f_{q, r}}(\alpha)\left(=(\bar{\lambda})^{1 / p}+\left(\lambda_{\infty}\right)^{1 / p}\right) .
\end{aligned}
$$

(ii) For fixed $p>1$, then there exists a unique number $q^{*}=q^{*}(p) \in$ $(0, p-1)$ such that

$$
\lim _{\alpha \rightarrow 1^{-}} T_{f_{q, r}}(\alpha)-\lim _{\alpha \rightarrow \infty} T_{f_{q, r}}(\alpha) \begin{cases}>0, & \text { if } q>q^{*}, \\ =0, & \text { if } q=q^{*} \\ <0, & \text { if } q<q^{*}\end{cases}
$$

(iii) $T_{f_{q, r}}(\alpha) \in C((0,1) \cup(1, \infty)), T_{f_{q, r}}(\alpha)$ is continuous at $1^{-}$, and $T_{f_{q, r}}(\alpha)$ is strictly increasing on $(0,1]$ and is strictly decreasing on (iv) $(1, \infty)$

$$
\begin{aligned}
& \lim _{\alpha \rightarrow 1^{+}} T_{f_{q, r}}^{\prime}(\alpha) \\
= & \begin{cases}-\infty, & \text { if } \max \{0,(p-2) / 2\}<q<p-1, \\
-2^{(2-p) / p}(p-1)^{1 / p} \frac{\Gamma\left(\frac{2}{p}\right) \Gamma\left(\frac{p-1}{p}\right)}{\Gamma\left(\frac{1}{p}\right)}, & \text { if } q=(p-2) / 2>0, \\
0, & \text { if } q<(p-2) / 2, p>2 .\end{cases}
\end{aligned}
$$


(III) If $r>p-1$, then

(i) $\lim _{\alpha \rightarrow 0^{+}} T_{f_{q, r}}(\alpha)=0, \lim _{\alpha \rightarrow \infty} T_{f_{q, r}}(\alpha)=0$,

$$
\lim _{\alpha \rightarrow 1^{-}} T_{f_{q, r}}(\alpha)=\left[\frac{(p-1)(q+1)}{p}\right]^{1 / p} \frac{p}{p-1-q} \equiv(\bar{\lambda})^{1 / p} \equiv T_{f_{q, r}}(1),
$$

and $\lim _{\alpha \rightarrow 1^{+}} T_{f_{q, r}}(\alpha)=\infty$.

(ii) $T_{f_{q, r}}(\alpha) \in C((0,1) \cup(1, \infty)), T_{f_{q, r}}(\alpha)$ is continuous at $1^{-}$, and $T_{f_{q, r}}(\alpha)$ is strictly increasing on $(0,1]$ and is strictly decreasing on $(1, \infty)$.

(B) If $q \geq p-1$, then

(I) If $r<p-1$, then

(i) $\lim _{\alpha \rightarrow 0^{+}} T_{f_{q, r}}(\alpha)=0$ and $\lim _{\alpha \rightarrow \infty} T_{f_{q, r}}(\alpha)=\infty$.

(ii) $\lim _{\alpha \rightarrow 1^{-}} T_{f_{q, r}}(\alpha)=\infty=\lim _{\alpha \rightarrow 1^{+}} T_{f_{q, r}}(\alpha)$.

(iii) $T_{f_{q, r}}(\alpha) \in C((0,1) \cup(1, \infty))$. Moreover, $T_{f_{q, r}}(\alpha)$ is strictly increasing on $(0,1)$ and has exactly one critical point at some $\alpha^{*}$, a local minimum, on $(1, \infty)$.

(II) If $r=p-1$, then

(i) $\lim _{\alpha \rightarrow 0^{+}} T_{f_{q, r}}(\alpha)=0$ and $\lim _{\alpha \rightarrow \infty} T_{f_{q, r}}(\alpha)=(p-1)^{1 / p} \frac{\pi}{p} \csc \frac{\pi}{p} \equiv$ $\left(\lambda_{\infty}\right)^{1 / p}$.

(ii) $\lim _{\alpha \rightarrow 1^{-}} T_{f_{q, r}}(\alpha)=\infty=\lim _{\alpha \rightarrow 1^{+}} T_{f_{q, r}}(\alpha)$.

(iii) $T_{f_{q, r}}(\alpha) \in C((0,1) \cup(1, \infty))$. Moreover, $T_{f_{q, r}}(\alpha)$ is strictly increasing on $(0,1)$ and is strictly decreasing on $(1, \infty)$.

(III) If $r>p-1$, then

(i) $\lim _{\alpha \rightarrow 0^{+}} T_{f_{q, r}}(\alpha)=0$ and $\lim _{\alpha \rightarrow \infty} T_{f_{q, r}}(\alpha)=0$.

(ii) $\lim _{\alpha \rightarrow 1^{-}} T_{f_{q, r}}(\alpha)=\infty=\lim _{\alpha \rightarrow 1^{+}} T_{f_{q, r}}(\alpha)$.

(iii) $T_{f_{q, r}}(\alpha) \in C((0,1) \cup(1, \infty))$. Moreover, $T_{f_{q, r}}(\alpha)$ is strictly increasing on $(0,1)$ and is strictly decreasing on $(1, \infty)$.

Remark 2. ([See Fig. 1(c1) and (c2)]). In Theorem 2.2(A)(I), if $q<r<p-1$, then $T_{f_{q, r}}(\alpha)$ is not necessarily a strictly increasing function of $\alpha$ on $[1, \infty)$. Actually, for any fixed $q<p-1$, if $r \rightarrow(p-1)^{-}$, by (3.13) stated below, we compute and find that

$$
T_{f_{q, r}}^{\prime}(\alpha=2) \rightarrow-(p-1)^{1 / p} \frac{1}{q+1} \int_{0}^{1} \frac{x^{\frac{-q}{q+1}}}{\left(1+\frac{p}{q+1} x\right)^{(p+1) / p}} d x<0 .
$$

So it is easy to see that $T_{f_{q, r}}(\alpha)$ has at least two critical points on $(1, \infty)$. Hence (1.1) has at least three positive solutions $u$ with $\|u\|_{\infty}>1$ for a certain range of $\lambda>0$. 
Remark 3. ([See Fig. 1(a)]). Numerical simulations suggest that, In Theorem 2.2(A)(I)(iv), if $r<q<p-1$, then $T_{f_{q, r}}(\alpha)$ has exactly one critical point at some $\alpha^{*}$, a local minimum, on $(1, \infty)$. But we are not able to give a proof.

Corollary 2.3. [See Figs. 1 and 2]). Consider (1.1) with constants $p>1$ and $q, r>0$. Then the following assertions $(A)-(H)$ hold:

(A) If $(\max \{0,(p-2) / 2\}<q=r<p-1)$ or $(r<q<p-1)$, then there exist two positive numbers

$$
\lambda^{*} \equiv\left[\min _{\alpha \in(1, \infty)} T_{f_{q, r}}(\alpha)\right]^{p}<\frac{(p-1)(q+1)}{p}\left(\frac{p}{p-1-q}\right)^{p}=\bar{\lambda}
$$

such that

(I) If $\max \{0,(p-2) / 2\}<q=r<p-1$, then $\lambda^{*}=\left[\min _{\alpha \in(1, \infty)} T_{f_{q, r}}(\alpha)\right]^{p}=$ $\left[T_{f_{q, r}}\left(\alpha^{*}\right)\right]^{p}$ and

(i) for $0<\lambda<\lambda^{*}$, (1.1) has exactly one positive solution $u$, which satisfies $\|u\|_{\infty}<1$

(ii) for $\lambda=\lambda^{*},(1.1)$ has exactly two positive solutions $u_{1}<u_{2}$, which satisfy $\left\|u_{1}\right\|_{\infty}<1<\left\|u_{2}\right\|_{\infty}$,

(iii) for $\lambda^{*}<\lambda<\bar{\lambda}$, (1.1) has exactly three positive solutions $u_{1}<u_{2}<$ $u_{3}$, which satisfy $\left\|u_{1}\right\|_{\infty}<1<\left\|u_{2}\right\|_{\infty}<\left\|u_{3}\right\|_{\infty}$,

(iv) for $\lambda=\bar{\lambda}$, (1.1) has exactly two positive solutions $u_{1}<u_{2}$, which satisfy $\left\|u_{1}\right\|_{\infty}=1<\left\|u_{2}\right\|_{\infty}$

(v) for $\lambda>\bar{\lambda}$, (1.1) has exactly one positive solution $u$, which satisfies $1<\|u\|_{\infty}$

(vi) For fixed $p>1$, the number $\lambda^{*}=\lambda^{*}(q)$ satisfies

$$
\begin{array}{ll}
\lim _{q \rightarrow(p-1)^{-}} \lambda^{*}(q)=(p-1)\left(\frac{\pi}{p} \csc \frac{\pi}{p}\right)^{p}=\lambda_{\infty}, & \text { if } p>1, \\
\lim _{q \rightarrow 0^{+}} \lambda^{*}(q)=\left(\frac{p}{p-1}\right)^{p-1}, & \text { if } 1<p \leq 2, \\
\lim _{q \rightarrow\left(\frac{p-2}{2}\right)^{+}} \lambda^{*}(q)=2^{p-1}(p-1), & \text { if } p \geq 2 .
\end{array}
$$

(II) If $r<q<p-1$, then

(i) for $0<\lambda<\lambda^{*}$, (1.1) has at least one positive solution $u$, which satisfies $\|u\|_{\infty}<1$,

(ii) for $\lambda=\lambda^{*},(1.1)$ has at least two positive solutions $u_{1}<u_{2}$, which satisfy $\left\|u_{1}\right\|_{\infty}<1<\left\|u_{2}\right\|_{\infty}$,

(iii) for $\lambda^{*}<\lambda<\bar{\lambda}$, (1.1) has at least three positive solutions $u_{1}<u_{2}<$ $u_{3}$, which satisfy $\left\|u_{1}\right\|_{\infty}<1<\left\|u_{2}\right\|_{\infty}<\left\|u_{3}\right\|_{\infty}$,

(iv) for $\lambda=\bar{\lambda}$, (1.1) has at least two positive solutions $u_{1}<u_{2}$, which satisfy $\left\|u_{1}\right\|_{\infty}=1<\left\|u_{2}\right\|_{\infty}$, 
(v) for $\lambda>\bar{\lambda}$, (1.1) has at least one positive solution u, which satisfies $1<\|u\|_{\infty}$.

(B) If $q=r=(p-2) / 2>0$, then for $\lambda>0$, (1.1) has exactly one positive solution.

(C) (I) If $q=r<(p-2) / 2$, then for $\lambda>0$, (1.1) has exactly one positive solution.

(II) If $q<r<p-1$, then for $\lambda>0$, (1.1) has at least one positive solution.

(D) If $q<r=p-1$, by (2.7), then

(I) If $q>q^{*}$, then

(i) for $0<\lambda \leq \lambda_{\infty}$, (1.1) has exactly one positive solution $u$, which satisfies $\|u\|_{\infty}<1$,

(ii) for $\lambda_{\infty}<\lambda \leq \bar{\lambda}$, (1.1) has exactly two positive solutions $u_{1}<u_{2}$, which satisfy $\left\|u_{1}\right\|_{\infty} \leq 1<\left\|u_{2}\right\|_{\infty}$,

(iii) for $\bar{\lambda}<\lambda<\left[(\bar{\lambda})^{1 / p}+\left(\lambda_{\infty}\right)^{1 / p}\right]^{p}$, (1.1) has exactly one positive solution $u$, which satisfies $\|u\|_{\infty}>1$,

(iv) for $\lambda \geq\left[(\bar{\lambda})^{1 / p}+\left(\lambda_{\infty}\right)^{1 / p}\right]^{p}$, (1.1) has no positive solution.

(II) If $q=q^{*}$, then

(i) for $0<\lambda \leq \lambda_{\infty}$, (1.1) has exactly one positive solution $u$, which satisfies $\|u\|_{\infty} \leq 1$,

(ii) for $\lambda_{\infty}<\lambda<\left[(\bar{\lambda})^{1 / p}+\left(\lambda_{\infty}\right)^{1 / p}\right]^{p}$, (1.1) has exactly one positive solution $u$, which satisfies $\|u\|_{\infty}>1$,

(iii) for $\lambda \geq\left[(\bar{\lambda})^{1 / p}+\left(\lambda_{\infty}\right)^{1 / p}\right]^{p}$, (1.1) has no positive solution.

(III) If $q<q^{*}$, then

(i) for $0<\lambda \leq \bar{\lambda}$, (1.1) has exactly one positive solution $u$, which satisfies $\|u\|_{\infty} \leq 1$

(ii) for $\bar{\lambda}<\lambda \leq \lambda_{\infty}$, (1.1) has no positive solution,

(iii) for $\lambda_{\infty}<\bar{\lambda}<\left[(\bar{\lambda})^{1 / p}+\left(\lambda_{\infty}\right)^{1 / p}\right]^{p}$, (1.1) has exactly one positive solution $u$, which satisfies $\|u\|_{\infty}>1$,

(iv) for $\lambda \geq\left[(\bar{\lambda})^{1 / p}+\left(\lambda_{\infty}\right)^{1 / p}\right]^{p}$, (1.1) has no positive solution.

(E) If $q<p-1<r$, then

(i) for $0<\lambda \leq \bar{\lambda}$, (1.1) has exactly two positive solutions $u_{1}<u_{2}$, which satisfy $\left\|u_{1}\right\|_{\infty} \leq 1<\left\|u_{2}\right\|_{\infty}$,

(ii) for $\lambda>\bar{\lambda}$, (1.1) has exactly one positive solution $u$, which satisfies $\|u\|_{\infty}>$ 1.

(F) If $q \geq p-1>r$, then there exists one positive number

$$
\lambda^{*} \equiv\left[\min _{\alpha \in(1, \infty)} T_{f_{q, r}}(\alpha)\right]^{p}=\left[T_{f_{q, r}}\left(\alpha^{*}\right)\right]^{p}
$$

such that 
(i) for $0<\lambda<\lambda^{*}$, (1.1) has exactly one positive solution $u$, which satisfies $\|u\|_{\infty}<1$

(ii) for $\lambda=\lambda^{*}$, (1.1) has exactly two positive solutions $u_{1}<u_{2}$, which satisfy $\left\|u_{1}\right\|_{\infty}<1<\left\|u_{2}\right\|_{\infty}$

(iii) for $\lambda>\lambda^{*}$, (1.1) has exactly three positive solutions $u_{1}<u_{2}<u_{3}$, which satisfy $\left\|u_{1}\right\|_{\infty}<1<\left\|u_{2}\right\|_{\infty}<\left\|u_{3}\right\|_{\infty}$.

(G) If $q \geq r=p-1$, then

(i) for $0<\lambda \leq \lambda_{\infty}$, (1.1) has exactly one positive solution $u$, which satisfies $\|u\|_{\infty}<1$

(ii) for $\lambda>\lambda_{\infty}$, (1.1) has exactly two positive solutions $u_{1}<u_{2}$, which satisfy $\left\|u_{1}\right\|_{\infty}<1<\left\|u_{2}\right\|_{\infty}$.

(H) If $q \geq p-1$ and $r>p-1$, then for $\lambda>0$, (1.1) has exactly two positive solutions $u_{1}<u_{2}$, which satisfy $\left\|u_{1}\right\|_{\infty}<1<\left\|u_{2}\right\|_{\infty}$.

\section{Proofs of Main Results}

To prove Theorems 2.1 and 2.2, we need the next Lemma 3.1 on properties of some special functions and on some formulas of some definite integrals. The proof of Lemma 3.1 is given in Section 4.

Lemma 3.1. The following identities hold:

(i)

$$
\Gamma(1+x)=x \Gamma(x) .
$$

(ii)

$$
\Gamma(x) \Gamma(1-x)=\frac{\pi}{\sin x \pi}(=\pi \csc x \pi) \quad(x \neq 0, \pm 1, \pm 2, \ldots) .
$$

(iii) For $0<a<1$ and $b>0$,

$$
\int_{0}^{1} \frac{x^{b-1}}{(1-x)^{a}} d x=\frac{\Gamma(b) \Gamma(1-a)}{\Gamma(1+b-a)} .
$$

(iv) For $1+\beta u>0$ and $b>0$,

$$
\int_{0}^{u} \frac{x^{b-1}}{(1+\beta x)^{a}} d x=\frac{u^{b}}{b} F(a, b ; 1+b ;-\beta u) .
$$

$(v)$

$$
F(a, b ; c ; x)=F(b, a ; c ; x) .
$$

(vi)

$$
F(a, b ; c ; 1)=\frac{\Gamma(c) \Gamma(c-a-b)}{\Gamma(c-a) \Gamma(c-b)} .
$$


(vii)

$$
F(0, b ; c ; x)=F(a, 0 ; c ; x)=F(a, b ; 0 ; x)=1 .
$$

(viii) For $|x| \rightarrow \infty$ and $b>a$,

$$
F(a, b ; c ; x) \sim \frac{\Gamma(c) \Gamma(b-a)}{\Gamma(c-a) \Gamma(b)(-x)^{a}}
$$

(ix) For $x<1$,

$$
F(a, b ; c ; x)=(1-x)^{-a} F\left(a, c-b ; c ; \frac{x}{x-1}\right) .
$$

(x) For $x<1$,

$$
F(a, b+1 ; c ; x)-F(a, b ; c ; x)=\frac{a x}{c} F(a+1, b+1 ; c+1 ; x) .
$$

$(x i)$

$$
\frac{d}{d x} F(a, b ; c ; x)=\frac{a b}{c} F(a+1, b+1 ; c+1 ; x) .
$$

(xii) For $x<1$,

$$
\begin{aligned}
& { }_{3} F_{2}\left(a_{1}, a_{2}+1, a_{3} ; b_{1}, b_{2} ; x\right)-{ }_{3} F_{2}\left(a_{1}, a_{2}, a_{3} ; b_{1}, b_{2} ; x\right) \\
= & \frac{a_{1} a_{3} x}{b_{1} b_{2}}{ }_{3} F_{2}\left(a_{1}+1, a_{2}+1, a_{3}+1 ; b_{1}+1, b_{2}+1 ; x\right) .
\end{aligned}
$$

(xiii) For $b_{2}-a_{1}>0$ and $b_{1}+b_{2}-a_{1}-a_{2}-a_{3}>0$,

$$
\begin{aligned}
& { }_{3} F_{2}\left(a_{1}, a_{2}, a_{3} ; b_{1}, b_{2} ; 1\right) \\
= & \frac{\Gamma\left(b_{2}\right) \Gamma\left(b_{1}+b_{2}-a_{1}-a_{2}-a_{3}\right)}{\Gamma\left(b_{2}-a_{1}\right) \Gamma\left(b_{1}+b_{2}-a_{2}-a_{3}\right)} \\
\times & { }_{3} F_{2}\left(a_{1}, b_{1}-a_{2}, b_{1}-a_{3} ; b_{1}, b_{1}+b_{2}-a_{2}-a_{3} ; 1\right) .
\end{aligned}
$$

(xiv)

$$
\begin{gathered}
\frac{d}{d x}{ }_{3} F_{2}\left(a_{1}, a_{2}, a_{3} ; b_{1}, b_{2} ; x\right)=\frac{a_{1} a_{2} a_{3}}{b_{1} b_{2}} \\
\times{ }_{3} F_{2}\left(a_{1}+1, a_{2}+1, a_{3}+1 ; b_{1}+1, b_{2}+1 ; x\right) .
\end{gathered}
$$

(xv) For $x \neq 0,-1,-2, \ldots$, the psi function

$$
\psi(x)=-\gamma-\sum_{j=0}^{\infty}\left(\frac{1}{x+j}-\frac{1}{j+1}\right) .
$$


(xvi) For $p>1, q>0$ with $q \neq p-1$, and $k \geq 1$,

$$
\begin{aligned}
& \int_{1}^{k} \frac{d t}{\left(t^{q+1}-1\right)^{1 / p}} \\
= & \frac{\Gamma\left(\frac{p-1}{p}\right) \Gamma\left(\frac{q+1-p}{p q+p}\right)}{(q+1) \Gamma\left(\frac{q}{q+1}\right)}-\frac{p k^{(p-1-q) / p}}{q+1-p} F\left(\frac{1}{p}, \frac{q+1-p}{p q+p} ; \frac{p q+q+1}{p q+p} ; k^{-(q+1)}\right) .
\end{aligned}
$$

(xvii) For $p>1$ and $k \geq 1$,

$$
\begin{aligned}
& \int_{1}^{k} \frac{d t}{\left(t^{p}-1\right)^{1 / p}} \\
= & \frac{1}{p^{2}}\left\{p\left[p \ln k-\gamma-\psi\left(\frac{p-1}{p}\right)\right]-k^{-p}{ }_{3} F_{2}\left(1,1, \frac{p+1}{p} ; 2,2 ; k^{-p}\right)\right\} .
\end{aligned}
$$

Proof of Theorem 2.1. (A) For $0<\alpha<1$,

(I) If $q \neq p-1$, by (1.5), we compute that

$$
\begin{aligned}
T_{f_{q, r}}(\alpha)= & \left(\frac{p-1}{p}\right)^{1 / p} \int_{0}^{\alpha} \frac{d u}{\left[F_{q, r}(\alpha)-F_{q, r}(u)\right]^{1 / p}} \\
= & {\left[\frac{(p-1)(q+1)}{p}\right]^{1 / p} \int_{0}^{\alpha} \frac{d u}{\left[(1-u)^{q+1}-(1-\alpha)^{q+1}\right]^{1 / p}} \text { (by (1.4)) } } \\
= & {\left[\frac{(p-1)(q+1)}{p}\right]^{1 / p}(1-\alpha)^{(p-1-q) / p} \int_{1}^{1 /(1-\alpha)} \frac{d t}{\left(t^{q+1}-1\right)^{1 / p}} } \\
& \left(\text { by setting } t=\frac{1-u}{1-\alpha}\right) \\
= & {\left[\frac{(p-1)(q+1)}{p}\right]^{1 / p}\left[\frac{\Gamma\left(\frac{p-1}{p}\right) \Gamma\left(\frac{q+1-p}{p q+p}\right)}{(q+1) \Gamma\left(\frac{q}{q+1}\right)}(1-\alpha)^{(p-1-q) / p}\right.} \\
& \left.\left.-\frac{p}{q+1-p} F\left(\frac{1}{p}, \frac{q+1-p}{p q+p} ; \frac{p q+q+1}{p q+p} ;(1-\alpha)^{q+1}\right]\right)\right]
\end{aligned}
$$

by Lemma 3.1(xvi).

(II) If $q=p-1$, we apply (3.3) and compute that

$$
\begin{aligned}
T_{f_{q, r}}(\alpha)= & (p-1)^{1 / p} \int_{1}^{1 /(1-\alpha)} \frac{d t}{\left(t^{p}-1\right)^{1 / p}} . \\
= & \frac{(p-1)^{1 / p}}{p^{2}}\left\{p\left[p \ln \left(\frac{1}{1-\alpha}\right)-\gamma-\psi\left(\frac{p-1}{p}\right)\right]\right. \\
& \left.-(1-\alpha)^{p}{ }_{3} F_{2}\left(1,1, \frac{p+1}{p} ; 2,2 ;(1-\alpha)^{p}\right)\right\}
\end{aligned}
$$


by Lemma 3.1(xvii).

(B) For $\alpha>1$, by (1.5), we compute that

$$
\begin{aligned}
& T_{f_{q, r}}(\alpha) \\
= & \left(\frac{p-1}{p}\right)^{1 / p} \int_{0}^{\alpha} \frac{d u}{\left[F_{q, r}(\alpha)-F_{q, r}(u)\right]^{1 / p}} \\
= & \left(\frac{p-1}{p}\right)^{1 / p}\left\{\int_{0}^{1} \frac{d u}{\left[F_{q, r}(\alpha)-F_{q, r}(u)\right]^{1 / p}}+\int_{1}^{\alpha} \frac{d u}{\left[F_{q, r}(\alpha)-F_{q, r}(u)\right]^{1 / p}}\right\} \\
= & \left(\frac{p-1}{p}\right)^{1 / p}\left\{\int_{0}^{1} \frac{d u}{\left[\frac{(\alpha-1)^{r+1}}{r+1}-\frac{(1-u)^{q+1}}{q+1}\right]^{1 / p}}+\int_{1}^{\alpha} \frac{d u}{\left[\frac{(\alpha-1)^{r+1}}{r+1}-\frac{(u-1)^{r+1}}{r+1}\right]^{1 / p}}\right\}
\end{aligned}
$$

(by (1.4))

$$
\begin{aligned}
= & {\left[\frac{(p-1)(r+1)}{p}\right]^{1 / p}\left\{\int_{0}^{1} \frac{d u}{\left[(\alpha-1)^{r+1}+\frac{r+1}{q+1}(1-u)^{q+1}\right]^{1 / p}}\right.} \\
& \left.+\int_{1}^{\alpha} \frac{d u}{\left[(\alpha-1)^{r+1}-(u-1)^{r+1}\right]^{1 / p}}\right\} \text { (by simple calculation) } \\
= & {\left[\frac{(p-1)(r+1)}{p}\right]^{1 / p}(\alpha-1)^{(p-1-r) / p} } \\
& \left\{\frac{1}{\alpha-1} \int_{0}^{1} \frac{d t}{\left[1+\frac{r+1}{q+1}(\alpha-1)^{-(r+1)} t^{q+1}\right]^{1 / p}}+\int_{0}^{1} \frac{d t}{\left(1-t^{r+1}\right)^{1 / p}}\right\}
\end{aligned}
$$

(by setting $t=\frac{1-u}{\alpha-1}$ in the first integral and $t=\frac{u-1}{\alpha-1}$ in the second integral)

$$
\begin{aligned}
= & {\left[\frac{(p-1)(r+1)}{p}\right]^{1 / p}(\alpha-1)^{(p-1-r) / p} } \\
& \left\{\frac{1}{\alpha-1} \int_{0}^{1} \frac{\frac{1}{q+1} x^{\frac{-q}{q+1}} d x}{\left[1+\frac{r+1}{q+1}(\alpha-1)^{-(r+1)} x\right]^{1 / p}}+\int_{0}^{1} \frac{\frac{1}{r+1} x^{\frac{-r}{r+1}} d x}{(1-x)^{1 / p}}\right\}
\end{aligned}
$$

(by setting $x=t^{q+1}$ in the first integral and $x=t^{r+1}$ in the second integral)

$$
=\left[\frac{(p-1)(r+1)}{p}\right]^{1 / p}(\alpha-1)^{(p-1-q) / p}\left\{\frac{1}{\alpha-1} F\left(\frac{1}{p}, \frac{1}{q+1} ; \frac{q+2}{q+1} ;-\frac{r+1}{q+1}(\alpha-1)^{-(r+1)}\right)\right.
$$




$$
\begin{aligned}
& \left.+\frac{\frac{1}{r+1} \Gamma\left(\frac{1}{r+1}\right) \Gamma\left(\frac{p-1}{p}\right)}{\Gamma\left(\frac{p r+2 p-r-1}{p r+p}\right)}\right\} \text { (by Lemma 3.1(iv) for the first integral and } \\
& \text { Lemma 3.1(iii) for the second integral) } \\
= & {\left[\frac{(p-1)(r+1)}{p}\right]^{1 / p}(\alpha-1)^{(p-1-q) / p} } \\
& \left\{\frac{1}{\alpha-1} F\left(\frac{1}{p}, \frac{1}{q+1} ; \frac{q+2}{q+1} ;-\frac{r+1}{q+1}(\alpha-1)^{-(r+1)}\right)+\frac{\Gamma\left(\frac{r+2}{r+1}\right) \Gamma\left(\frac{p-1}{p}\right)}{\Gamma\left(\frac{p r+2 p-r-1}{p r+p}\right)}\right\}
\end{aligned}
$$

by applying Lemma 3.1(i). The proof of Theorem 2.1 is complete.

Proof of Theorem 2.2. (A) Suppose $q<p-1$.

(I) (i) If $r<p-1$, then the results $\lim _{\alpha \rightarrow 0^{+}} T_{f_{q, r}}(\alpha)=0, \lim _{\alpha \rightarrow \infty} T_{f_{q, r}}(\alpha)=\infty$, and $\lim _{\alpha \rightarrow 1^{-}} T_{f_{q, r}}(\alpha)=\left[\frac{(p-1)(q+1)}{p}\right]^{1 / p} \frac{p}{p-1-q}\left(\equiv T_{f_{q, r}}(1)\right)$ follow by Proposition 1.1(ii), (iv), and (iii), respectively. We prove (2.5) by Theorem 2.1(B). We compute that

$$
\begin{aligned}
& \lim _{\alpha \rightarrow 1^{+}} T_{f_{q, r}}(\alpha) \\
= & \lim _{\alpha \rightarrow 1^{+}}\left\{\left[\frac{(p-1)(r+1)}{p}\right]^{1 / p}(\alpha-1)^{(p-1-r) / p}\right. \\
\times & {\left.\left[\frac{1}{\alpha-1} F\left(\frac{1}{p}, \frac{1}{q+1} ; \frac{q+2}{q+1} ;-\frac{r+1}{q+1}(\alpha-1)^{-(r+1)}\right)+\frac{\Gamma\left(\frac{r+2}{r+1}\right) \Gamma\left(\frac{p-1}{p}\right)}{\Gamma\left(\frac{p r+2 p-r-1}{p r+p}\right)}\right]\right\} } \\
= & \lim _{\alpha \rightarrow 1^{+}}\left\{\left[\frac{(p-1)(r+1)}{p}\right]^{1 / p}(\alpha-1)^{(p-1-r) / p}\right. \\
& {\left[\frac{\left.\Gamma\left(\frac{r+2}{r+1}\right) \Gamma\left(\frac{p-1}{p}\right)\right]}{\Gamma\left(\frac{p q+2 p-q-1}{p q+p}\right) \Gamma\left(\frac{1}{q+1}\right)(\alpha-1)^{(p-1-r) / p}}\left(\frac{q+2}{q+1}\right) \Gamma\left(\frac{p-1-q}{p q+p}\right)\right.} \\
= & \lim _{\alpha \rightarrow 1^{+}}\left\{[ \frac { ( p - 1 ) ( r + 1 ) } { p } ] ^ { 1 / p } \left[\frac{\left(\frac{q+1}{r+1}\right)^{1 / p} \Gamma\left(\frac{q+2}{q+1}\right) \Gamma\left(\frac{p-1-q}{p q+p}\right)}{\Gamma\left(\frac{p q+2 p-q-1}{p q+p}\right) \Gamma\left(\frac{1}{q+1}\right)}\right.\right. \\
& \left.\left.+\frac{\Gamma\left(\frac{r+2}{r+1}\right) \Gamma\left(\frac{p-1}{p}\right)}{\Gamma\left(\frac{p r+2 p-r-1}{p r+p}\right)}(\alpha-1)^{(p-1-r) / p}\right]\right\} \text { (by simple calculation) }
\end{aligned}
$$




$$
=\left[\frac{(p-1)(r+1)}{p}\right]^{1 / p} \frac{\left(\frac{q+1}{r+1}\right)^{1 / p} \Gamma\left(\frac{q+2}{q+1}\right) \Gamma\left(\frac{p-1-q}{p q+p}\right)}{\Gamma\left(\frac{p q+2 p-q-1}{p q+p}\right) \Gamma\left(\frac{1}{q+1}\right)}
$$

(since $\lim _{\alpha \rightarrow 1^{+}}(\alpha-1)^{(p-1-r) / p}=0$ for $r<p-1$ )

$$
\begin{aligned}
& =\left[\frac{(p-1)(q+1)}{p}\right]^{1 / p} \frac{\frac{1}{q+1} \Gamma\left(\frac{1}{q+1}\right) \Gamma\left(\frac{p-1-q}{p q+p}\right)}{\frac{p-1-q}{p q+q} \Gamma\left(\frac{p-1-q}{p q+p}\right) \Gamma\left(\frac{1}{q+1}\right)}(\text { by Lemma 3.1(i)) } \\
& =\left[\frac{(p-1)(q+1)}{p}\right]^{1 / p} \frac{p}{p-1-q}=T_{f_{q, r}}(1)=\lim _{\alpha \rightarrow 1^{-}} T_{f_{q, r}}(\alpha)
\end{aligned}
$$

by Proposition 1.1(iii).

(I) (ii) For $r<p-1$, we obtain that $T_{f_{q, r}}(\alpha) \in C(0, \infty)$ and $T_{f_{q, r}}(\alpha)$ is strictly increasing on $(0,1]$ by Proposition 1.1(i) and (3.5).

(I) (iii) For $r<p-1$ and $\alpha>1$, by Theorem 2.1(B), we compute that

$$
\begin{aligned}
& T_{f_{q, r}}^{\prime}(\alpha) \\
& =\frac{d}{d \alpha}\left\{\left[\frac{(p-1)(r+1)}{p}\right]^{1 / p}(\alpha-1)^{(p-1-r) / p}\right. \\
& \left.\times\left[\frac{1}{\alpha-1} F\left(\frac{1}{p}, \frac{1}{q+1} ; \frac{q+2}{q+1} ;-\frac{r+1}{q+1}(\alpha-1)^{-(r+1)}+\frac{\Gamma\left(\frac{r+2}{r+1}\right) \Gamma\left(\frac{p-1}{p}\right)}{\Gamma\left(\frac{p r+2 p-r-1}{p r+p}\right)}\right)\right]\right\} \\
& =\left[\frac{(p-1)(r+1)}{p}\right]^{1 / p} \\
& \times\left\{-\frac{r+1}{p} F\left(\frac{1}{p}, \frac{1}{q+1} ; \frac{q+2}{q+1} ;-\frac{r+1}{q+1}(\alpha-1)^{-(r+1)}\right)(\alpha-1)^{-(p+1+r) / p}\right. \\
& +\frac{p-1-r}{p} \frac{\Gamma\left(\frac{r+2}{r+1}\right) \Gamma\left(\frac{p-1}{p}\right)}{\Gamma\left(\frac{p r+2 p-r-1}{p r+p}\right)}(\alpha-1)^{-(r+1) / p}+\frac{(r+1)^{2}}{p(q+2)(q+1)} \\
& \left.F\left(\frac{p+1}{p}, \frac{q+2}{q+1} ; \frac{2 q+3}{q+1} ;-\frac{r+1}{q+1}(\alpha-1)^{-(r+1)}\right)(\alpha-1)^{\frac{-p-1-r}{p}-r-1}\right\}
\end{aligned}
$$

(by Lemma 3.1(xi))

$$
\begin{aligned}
& =\left[\frac{(p-1)(r+1)}{p}\right]^{1 / p} \\
& \times\left\{-\frac{r+1}{p} F\left(\frac{1}{p}, \frac{1}{q+1} ; \frac{q+2}{q+1} ;-\frac{r+1}{q+1}(\alpha-1)^{-(r+1)}\right)(\alpha-1)^{-(p+1+r) / p}\right.
\end{aligned}
$$




$$
\begin{aligned}
+ & \frac{p-1-r}{p} \frac{\Gamma\left(\frac{r+2}{r+1}\right) \Gamma\left(\frac{p-1}{p}\right)}{\Gamma\left(\frac{p r+2 p-r-1}{p r+p}\right)}(\alpha-1)^{-(r+1) / p} \\
+ & \frac{r+1}{q+1}\left[F\left(\frac{1}{p}, \frac{1}{q+1} ; \frac{q+2}{q+1} ;-\frac{r+1}{q+1}(\alpha-1)^{-(r+1)}\right)(\alpha-1)^{-(p+1+r) / p}\right. \\
& \left.\left.-F\left(\frac{1}{p}, \frac{q+2}{q+1} ; \frac{q+2}{q+1} ;-\frac{r+1}{q+1}(\alpha-1)^{-(r+1)}\right)\right]\right\}(\text { by Lemma 3.1(x)) } \\
= & {\left[\frac{(p-1)(r+1)}{p}\right]^{1 / p} } \\
\times\{- & \frac{r+1}{p} F\left(\frac{1}{p}, \frac{1}{q+1} ; \frac{q+2}{q+1} ;-\frac{r+1}{q+1}(\alpha-1)^{-(r+1)}\right)(\alpha-1)^{-(p+1+r) / p} \\
& +\frac{p-1-r}{p} \frac{\Gamma\left(\frac{r+2}{r+1}\right) \Gamma\left(\frac{p-1}{p}\right)}{\Gamma\left(\frac{p r+2 p-r-1}{p r+p}\right)}(\alpha-1)^{-(r+1) / p} \\
& +\frac{r+1}{q+1}\left[F\left(\frac{1}{p}, \frac{1}{q+1} ; \frac{q+2}{q+1} ;-\frac{r+1}{q+1}(\alpha-1)^{-(r+1)}\right)(\alpha-1)^{-(p+1+r) / p}\right. \\
- & \left.\left.F\left(\frac{1}{p}, 0 ; \frac{q+2}{q+1} ; \frac{w}{w-1}\right)\left(1+\frac{r+1}{q+1}(\alpha-1)^{-(r+1)}\right)^{-1 / p}\right]\right\}
\end{aligned}
$$

(by Lemma 3.1(ix) for the last term where $w \equiv-\frac{r+1}{q+1}(\alpha-1)^{-(r+1)}$ )

$$
\begin{aligned}
= & {\left[\frac{(p-1)(r+1)}{p}\right]^{1 / p} } \\
\times & \left\{-\frac{r+1}{p} F\left(\frac{1}{p}, \frac{1}{q+1} ; \frac{q+2}{q+1} ;-\frac{r+1}{q+1}(\alpha-1)^{-(r+1)}\right)(\alpha-1)^{-(p+1+r) / p}\right. \\
& +\frac{p-1-r}{p} \frac{\Gamma\left(\frac{r+2}{r+1}\right) \Gamma\left(\frac{p-1}{p}\right)}{\Gamma\left(\frac{p r+2 p-r-1}{p r+p}\right)}(\alpha-1)^{-(r+1) / p} \\
& +\frac{r+1}{q+1}\left[F\left(\frac{1}{p}, \frac{1}{q+1} ; \frac{q+2}{q+1} ;-\frac{r+1}{q+1}(\alpha-1)^{-(r+1)}\right)(\alpha-1)^{-(p+1+r) / p}\right. \\
& \left.\left.-\left(1+\frac{r+1}{q+1}(\alpha-1)^{-(r+1)}\right)^{-1 / p}\right]\right\}(\text { by Lemma 3.1(vii) for the last term }) \\
= & \left.\frac{(p-1)(r+1)}{p}\right]^{1 / p}\left\{\frac{p-1-r}{p} \frac{\Gamma\left(\frac{r+2}{r+1}\right) \Gamma\left(\frac{p-1}{p}\right)}{\Gamma\left(\frac{p r+2 p-r-1}{p r+p}\right)}(\alpha-1)^{-(r+1) / p}\right. \\
& +\frac{(p-1-q)(r+1)}{p q+q} F\left(\frac{1}{p}, \frac{1}{q+1} ; \frac{q+2}{q+1} ;-\frac{r+1}{q+1}(\alpha-1)^{-(r+1)}\right) \\
& (\alpha-1)^{-(p+1+r) / p}
\end{aligned}
$$




$$
\left.-\frac{r+1}{q+1}\left(1+\frac{r+1}{q+1}(\alpha-1)^{-(r+1)}\right)^{-1 / p}(\alpha-1)^{-(p+1+r) / p}\right\}
$$

(by simple calculation)

$$
\begin{aligned}
= & {\left[\frac{(p-1)(r+1)}{p}\right]^{1 / p}\left\{\frac{p-1-r}{p} \frac{\Gamma\left(\frac{r+2}{r+1}\right) \Gamma\left(\frac{p-1}{p}\right)}{\Gamma\left(\frac{p r+2 p-r-1}{p r+p}\right)}(\alpha-1)^{-(r+1) / p}\right.} \\
& \left.-\frac{r+1}{p} F\left(\frac{p+1}{p}, \frac{1}{q+1} ; \frac{q+2}{q+1} ;-\frac{r+1}{q+1}(\alpha-1)^{-(r+1)}\right)(\alpha-1)^{-(p+1+r) / p}\right\}
\end{aligned}
$$

by showing that

$$
\begin{aligned}
& \frac{(p-1-q)(r+1)}{p q+q} F\left(\frac{1}{p}, \frac{1}{q+1} ; \frac{q+2}{q+1} ;-\frac{r+1}{q+1}(\alpha-1)^{-(r+1)}\right) \\
& -\frac{r+1}{q+1}\left(1+\frac{r+1}{q+1}(\alpha-1)^{-(r+1)}\right)^{-1 / p} \\
= & -\frac{r+1}{p} F\left(\frac{p+1}{p}, \frac{1}{q+1} ; \frac{q+2}{q+1} ;-\frac{r+1}{q+1}(\alpha-1)^{-(r+1)}\right) .
\end{aligned}
$$

The proof of (3.8) is easy but tedious; we omit it. Then by (3.7), we compute that

$$
\begin{aligned}
& \lim _{\alpha \rightarrow 1^{+}} T_{f_{q, r}}^{\prime}(\alpha) \\
= & \lim _{\alpha \rightarrow 1^{+}}\left\{[ \frac { ( p - 1 ) ( r + 1 ) } { p } ] ^ { 1 / p } \left[\frac{p-1-r}{p} \frac{\Gamma\left(\frac{r+2}{r+1}\right) \Gamma\left(\frac{p-1}{p}\right)}{\Gamma\left(\frac{p r+2 p-r-1}{p r+p}\right)}(\alpha-1)^{-(r+1) / p}\right.\right. \\
& \left.\left.-\frac{r+1}{p} F\left(\frac{p+1}{p}, \frac{1}{q+1} ; \frac{q+2}{q+1} ;-\frac{r+1}{q+1}(\alpha-1)^{-(r+1)}\right)(\alpha-1)^{-(p+1+r) / p}\right]\right\} \\
= & \lim _{\alpha \rightarrow 1^{+}}\left\{[ \frac { ( p - 1 ) ( r + 1 ) } { p } ] ^ { 1 / p } \left[\frac{p-1-r}{p} \frac{\Gamma\left(\frac{r+2}{r+1}\right) \Gamma\left(\frac{p-1}{p}\right)}{\Gamma\left(\frac{p r+2 p-r-1}{p r+p}\right)}(\alpha-1)^{-(r+1) / p}\right.\right. \\
& \left.\left.-\frac{r+1}{p} F\left(\frac{1}{q+1}, \frac{p+1}{p} ; \frac{q+2}{q+1} ;-\frac{r+1}{q+1}(\alpha-1)^{-(r+1)}\right)(\alpha-1)^{-(p+1+r) / p}\right]\right\}
\end{aligned}
$$

(by Lemma 3.1(v))

$$
\begin{aligned}
= & \lim _{\alpha \rightarrow 1^{+}}\left\{[ \frac { ( p - 1 ) ( r + 1 ) } { p } ] ^ { 1 / p } \left[\frac{p-1-r}{p} \frac{\Gamma\left(\frac{r+2}{r+1}\right) \Gamma\left(\frac{p-1}{p}\right)}{\Gamma\left(\frac{p r+2 p-r-1}{p r+p}\right)}(\alpha-1)^{-(r+1) / p}\right.\right. \\
& \left.\left.-\frac{r+1}{p}\left(\frac{r+1}{q+1}\right)^{-\frac{1}{q+1}} \frac{\Gamma\left(\frac{q+2}{q+1}\right) \Gamma\left(\frac{p q+q+1}{p q+p}\right)}{\Gamma(1) \Gamma\left(\frac{p+1}{p}\right)}(\alpha-1)^{\frac{-r-1}{p}+\frac{r-q}{q+1}}\right]\right\}
\end{aligned}
$$

(by Lemma 3.1(viii))

$$
=\lim _{\alpha \rightarrow 1^{+}}\left\{[ \frac { ( p - 1 ) ( r + 1 ) } { p } ] ^ { 1 / p } \left[\frac{p-1-r}{p} \frac{\Gamma\left(\frac{r+2}{r+1}\right) \Gamma\left(\frac{p-1}{p}\right)}{\Gamma\left(\frac{p r+2 p-r-1}{p r+p}\right)}(\alpha-1)^{-(r+1) / p}\right.\right.
$$




$$
\left.\left.-\frac{r+1}{p}\left(\frac{r+1}{q+1}\right)^{-\frac{1}{q+1}} \frac{\frac{1}{q+1} \Gamma\left(\frac{1}{q+1}\right) \Gamma\left(\frac{p q+q+1}{p q+p}\right)}{\frac{1}{p} \Gamma\left(\frac{1}{p}\right)}(\alpha-1)^{\frac{-r-1}{p}+\frac{r-q}{q+1}}\right]\right\}
$$

(by Lemma 3.1(i))

$$
\begin{aligned}
= & \lim _{\alpha \rightarrow 1^{+}}\left\{[ \frac { ( p - 1 ) ( r + 1 ) } { p } ] ^ { 1 / p } \left[\frac{p-1-r}{p} \frac{\Gamma\left(\frac{r+2}{r+1}\right) \Gamma\left(\frac{p-1}{p}\right)}{\Gamma\left(\frac{p r+2 p-r-1}{p r+p}\right)}(\alpha-1)^{-(r+1) / p}\right.\right. \\
& \left.\left.-\left(\frac{r+1}{q+1}\right)^{\frac{q}{q+1}} \frac{\Gamma\left(\frac{1}{q+1}\right) \Gamma\left(\frac{p q+q+1}{p q+p}\right)}{\Gamma\left(\frac{1}{p}\right)}(\alpha-1)^{\frac{-r-1}{p}+\frac{r-q}{q+1}}\right]\right\}
\end{aligned}
$$

(by simple calculation)

$$
\begin{aligned}
= & \lim _{\alpha \rightarrow 1^{+}}\left\{[ \frac { ( p - 1 ) ( r + 1 ) } { p } ] ^ { 1 / p } ( \alpha - 1 ) ^ { - ( r + 1 ) / p } \left[\frac{p-1-r}{p} \frac{\Gamma\left(\frac{r+2}{r+1}\right) \Gamma\left(\frac{p-1}{p}\right)}{\Gamma\left(\frac{p r+2 p-r-1}{p r+p}\right)}\right.\right. \\
& \left.\left.-\left(\frac{r+1}{q+1}\right)^{\frac{q}{q+1}} \frac{\Gamma\left(\frac{1}{q+1}\right) \Gamma\left(\frac{p q+q+1}{p q+p}\right)}{\Gamma\left(\frac{1}{p}\right)}(\alpha-1)^{\frac{r-q}{q+1}}\right]\right\}
\end{aligned}
$$

(by simple calculation)

$$
=\left\{\begin{array}{c}
-\infty, \text { if }(\max \{0,(p-2) / 2\}<q=r<p-1) \text { or }(r<q<p-1), \\
0, \text { if } q=r=(p-2) / 2>0, \\
\infty, \quad \text { if }(q=r<(p-2) / 2 \text { and } p>2) \text { or }(q<r<p-1),
\end{array}\right.
$$

by some analysis of (3.10). The proof of (3.11) is easy but tedious; we omit it.

(I) (iv) For $r<q<p-1, T_{f_{q, r}}(\alpha)$ has at least one critical point at some $\alpha^{*}$, a local minimum, on $(1, \infty)$ since $\lim _{\alpha \rightarrow 1^{+}} T_{f_{q, r}}^{\prime}(\alpha)=-\infty, \lim _{\alpha \rightarrow \infty} T_{f_{q, r}}(\alpha)=\infty$ and $T_{f_{q, r}}(\alpha)$ is continuous on $(0, \infty)$. The rest part of (I)(iv) for $\max \{0,(p-2) / 2\}<$ $q=r<p-1$ is from [4, Theorem 2.1(A)(iv)].

(I) (v) This part for $q=r \leq(p-2) / 2$ and $p>2$ is from [4, Theorem 2.1(A)(v)].

(II) (i) If $r=p-1$, then the results $\lim _{\alpha \rightarrow 0^{+}} T_{f_{q, r}}(\alpha)=0, \lim _{\alpha \rightarrow \infty} T_{f_{q, r}}(\alpha)=$ $(p-1)^{1 / p} \frac{\pi}{p} \csc \frac{\pi}{p}$, and $\lim _{\alpha \rightarrow 1^{-}} T_{f_{q, r}}(\alpha)=\left[\frac{(p-1)(q+1)}{p}\right]^{1 / p} \frac{p}{p-1-q}\left(\equiv T_{f_{q, r}}(1)\right)$ follow by Proposition 1.1(ii), (iv), and (iii), respectively. In addition, by applying (3.4), we compute that

$$
\begin{aligned}
& \lim _{\alpha \rightarrow 1^{+}} T_{f_{q, r}}(\alpha) \\
= & (p-1)^{1 / p}\left[\frac{\left(\frac{q+1}{p}\right)^{1 / p} \Gamma\left(\frac{q+2}{q+1}\right) \Gamma\left(\frac{p-1-q}{p q+p}\right)}{\Gamma\left(\frac{p q+2 p-q-1}{p q+p}\right) \Gamma\left(\frac{1}{q+1}\right)}+\Gamma\left(\frac{p+1}{p}\right) \Gamma\left(\frac{p-1}{p}\right)\right] \\
= & (p-1)^{1 / p}\left[\frac{\left(\frac{q+1}{p}\right)^{1 / p} \frac{1}{q+1} \Gamma\left(\frac{1}{q+1}\right) \Gamma\left(\frac{p-1-q}{p q+p}\right)}{\left(\frac{p-1-q}{p q+p}\right) \Gamma\left(\frac{p-1-q}{p q+p}\right) \Gamma\left(\frac{1}{q+1}\right)}+\frac{1}{p} \Gamma\left(\frac{1}{p}\right) \Gamma\left(\frac{p-1}{p}\right)\right]
\end{aligned}
$$


(by Lemma 3.1(i))

$=\left[\frac{(p-1)(q+1)}{p}\right]^{1 / p} \frac{p}{p-1-q}+(p-1)^{1 / p} \frac{1}{p} \Gamma\left(\frac{1}{p}\right) \Gamma\left(\frac{p-1}{p}\right)$

(by simple calculation)

$$
\begin{aligned}
& =\left[\frac{(p-1)(q+1)}{p}\right]^{1 / p} \frac{p}{p-1-q}+(p-1)^{1 / p} \frac{\pi}{p} \csc \frac{\pi}{p} \text { (by Lemma 3.1(ii)) } \\
& =\lim _{\alpha \rightarrow 1^{-}} T_{f_{q, r}}(\alpha)+\lim _{\alpha \rightarrow \infty} T_{f_{q, r}}(\alpha) .
\end{aligned}
$$

(II) (ii) If $r=p-1$, let

$$
\begin{aligned}
G(p, q) & \equiv \lim _{\alpha \rightarrow 1^{-}} T_{f_{q, r}}(\alpha)-\lim _{\alpha \rightarrow \infty} T_{f_{q, r}}(\alpha) \\
& =\left[\frac{(p-1)(q+1)}{p}\right]^{1 / p} \frac{p}{p-1-q}-(p-1)^{1 / p} \frac{\pi}{p} \csc \frac{\pi}{p}
\end{aligned}
$$

which is easy to see that it is strictly increasing in $q>0$. Also, for $q=0$ and $p>1$,

$$
G(p, 0)=\left(\frac{p-1}{p}\right)^{1 / p} \frac{p}{p-1}-(p-1)^{1 / p} \frac{\pi}{p} \csc \frac{\pi}{p}<0 ;
$$

see Fig. 3. Note that $\lim _{p \rightarrow 1^{+}} G(p, 0)=\lim _{p \rightarrow \infty} G(p, 0)=0$.

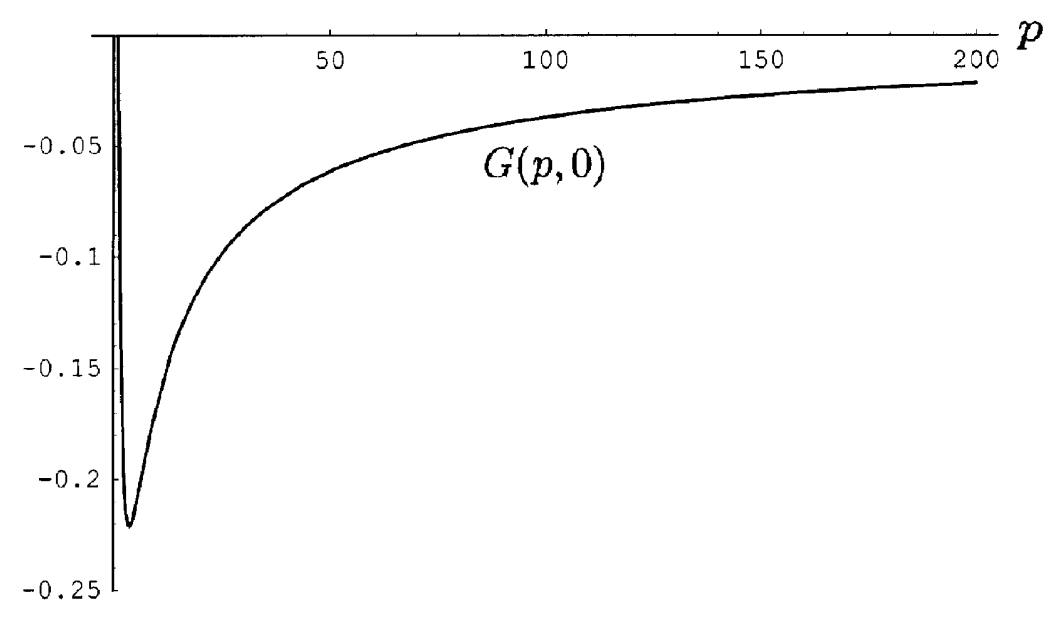

Fig. 3. Graph of $G(p, 0)=\left(\frac{p-1}{p}\right)^{1 / p} \frac{p}{p-1}-(p-1)^{1 / p} \frac{\pi}{p} \csc \frac{\pi}{p}$ on $(1,200)$.

In addition, by (3.12), for fixed $p>1$, we compute that 


$$
\begin{aligned}
& \lim _{q \rightarrow(p-1)^{-}} G(p, q) \\
= & \lim _{q \rightarrow(p-1)^{-}}\left\{\left[\frac{(p-1)(q+1)}{p}\right]^{1 / p} \frac{p}{p-1-q}-(p-1)^{1 / p} \frac{\pi}{p} \csc \frac{\pi}{p}\right\}=\infty .
\end{aligned}
$$

So, by applying the Intermediate Value Theorem, for fixed $p>1$, there exists a unique $q^{*}=q^{*}(p) \in(0, p-1)$ such that

$$
G(p, q)=\lim _{\alpha \rightarrow 1^{-}} T_{f_{q, r}}(\alpha)-\lim _{\alpha \rightarrow \infty} T_{f_{q, r}}(\alpha) \begin{cases}>0, & \text { if } q>q^{*} \\ =0, & \text { if } q=q^{*} \\ <0, & \text { if } q<q^{*}\end{cases}
$$

(II) (iii) For $r=p-1$, the results $T_{f_{q, r}}(\alpha) \in C((0,1) \cup(1, \infty))$ and $T_{f_{q, r}}(\alpha)$ is strictly increasing on $(0,1]$ follow by Proposition 1.1(i). For $r=p-1$ and $\alpha>1$, by (3.7), we compute that

$$
\begin{aligned}
& T_{f_{q, r}}^{\prime}(\alpha) \\
= & -(p-1)^{1 / p} F\left(\frac{p+1}{p}, \frac{1}{q+1} ; \frac{q+2}{q+1} ;-\frac{p}{q+1}\left(\frac{1}{\alpha-1}\right)^{p}\right)_{-q}^{p}(\alpha-1)^{-2} \\
= & -(p-1)^{1 / p} \frac{1}{q+1}(\alpha-1)^{\frac{p-2 q-2}{q+1}} \int_{0}^{(\alpha-1)^{-(r+1)}} \frac{x^{\frac{-q}{q+1}}}{\left(1+\frac{p}{q+1} x\right)^{(p+1) / p}} d x \\
& \quad \text { (by Lemma 3.1(iv)) } \\
< & 0
\end{aligned}
$$

for $\alpha>1$. Hence, for $r=p-1, T_{f_{q, r}}(\alpha)$ is strictly decreasing on $(1, \infty)$.

(II) (iv) For $r=p-1$, by (3.10), we compute that

$$
\begin{aligned}
& \lim _{\alpha \rightarrow 1^{+}} T_{f_{q, r}}^{\prime}(\alpha) \\
= & \lim _{\alpha \rightarrow 1^{+}}\left[-(p-1)^{1 / p}\left(\frac{p}{q+1}\right)^{\frac{q}{q+1}} \frac{\Gamma\left(\frac{1}{q+1}\right) \Gamma\left(\frac{p q+q+1}{p q+p}\right)}{\Gamma\left(\frac{1}{p}\right)}(\alpha-1)^{\frac{p-2 q-2}{q+1}}\right] \\
= & \begin{array}{ll}
-\infty, & \text { if } \max \{0,(p-2) / 2\}<q<p-1, \\
-2^{(2-p) / p}(p-1)^{1 / p} \frac{\Gamma\left(\frac{2}{p}\right) \Gamma\left(\frac{p-1}{p}\right)}{\Gamma\left(\frac{1}{p}\right)}, & \text { if } q=(p-2) / 2>0, \\
0, & \text { if } q<(p-2) / 2, \text { and } p>2 .
\end{array}
\end{aligned}
$$

by simple calculation.

(III) (i) If $r>p-1$, then the results $\lim _{\alpha \rightarrow 0^{+}} T_{f_{q, r}}(\alpha)=0, \lim _{\alpha \rightarrow \infty} T_{f_{q, r}}(\alpha)=0$, and $\lim _{\alpha \rightarrow 1^{-}} T_{f_{q, r}}(\alpha)=\left[\frac{(p-1)(q+1)}{p}\right]^{1 / p} \frac{p}{p-1-q}\left(\equiv T_{f_{q, r}}(1)\right)$ follow by Proposition 1.1(ii), (iv), and (iii), respectively. In addition, by applying (3.4), we compute that 


$$
\begin{aligned}
& \lim _{\alpha \rightarrow 1^{+}} T_{f_{q, r}}(\alpha) \\
= & \lim _{\alpha \rightarrow 1^{+}}\left\{[ \frac { ( p - 1 ) ( r + 1 ) } { p } ] ^ { 1 / p } \left[\frac{\left(\frac{q+1}{r+1}\right)^{1 / p} \Gamma\left(\frac{q+2}{q+1}\right) \Gamma\left(\frac{p-1-q}{p q+p}\right)}{\Gamma\left(\frac{p q+2 p-q-1}{p q+p}\right) \Gamma\left(\frac{1}{q+1}\right)}\right.\right. \\
& \left.\left.+\frac{\Gamma\left(\frac{r+2}{r+1}\right) \Gamma\left(\frac{p-1}{p}\right)}{\Gamma\left(\frac{p r+2 p-r-1}{p r+p}\right)}(\alpha-1)^{(p-1-r) / p}\right]\right\}
\end{aligned}
$$

(III) (ii) For $r>p-1$, the results $T_{f_{q, r}}(\alpha) \in C((0,1) \cup(1, \infty))$ and $T_{f_{q, r}}(\alpha)$ is strictly increasing on $(0,1]$ follow by Proposition 1.1(i). For $\alpha>1$, by (3.7), we compute that

$$
\begin{aligned}
& T_{f_{q, r}}^{\prime}(\alpha) \\
= & {\left[\frac{(p-1)(r+1)}{p}\right]^{1 / p}\left\{\frac{p-1-r}{p} \frac{\Gamma\left(\frac{r+2}{r+1}\right) \Gamma\left(\frac{p-1}{p}\right)}{\Gamma\left(\frac{p r+2 p-r-1}{p r+p}\right)}(\alpha-1)^{-(r+1) / p}\right.} \\
& \left.-\frac{r+1}{p} F\left(\frac{p+1}{p}, \frac{1}{q+1} ; \frac{q+2}{q+1} ;-\frac{r+1}{q+1}(\alpha-1)^{-(r+1)}\right)(\alpha-1)^{-(p+1+r) / p}\right\} \\
= & {\left[\frac{(p-1)(r+1)}{p}\right]^{1 / p}(\alpha-1)^{-(r+1) / p}\left\{\frac{p-1-r}{p} \frac{\Gamma\left(\frac{r+2}{r+1}\right) \Gamma\left(\frac{p-1}{p}\right)}{\Gamma\left(\frac{p r+2 p-r-1}{p r+p}\right)}\right.} \\
& \left.-\frac{r+1}{p q+p}(\alpha-1)^{\frac{r-q}{q+1}} \int_{0}^{(\alpha-1)^{-(r+1)}} \frac{x^{\frac{-q}{q+1}}}{\left(1+\frac{r+1}{q+1} x\right)^{\frac{p+1}{p}}} d x\right\} \\
< & 0
\end{aligned}
$$

since $r>p-1$. Hence $T_{f_{q, r}}(\alpha)$ is strictly decreasing on $(1, \infty)$.

(B) Suppose $q \geq p-1$.

(I) (i) If $r<p-1$, then the results $\lim _{\alpha \rightarrow 0^{+}} T_{f_{q, r}}(\alpha)=0$ and $\lim _{\alpha \rightarrow \infty} T_{f_{q, r}}(\alpha)=$ $\infty$ follow by Proposition 1.1(ii) and (iv), respectively.

(I) (ii) By Proposition 1.1(iii), $\lim _{\alpha \rightarrow 1^{-}} T_{f_{q, r}}(\alpha)=\infty$. In addition, for $r<p-1$ and $\alpha>1$, by Theorem 2.1(B), we compute that

$$
\begin{aligned}
& \lim _{\alpha \rightarrow 1^{+}} T_{f_{q, r}}(\alpha) \\
= & \lim _{\alpha \rightarrow 1^{+}}\left\{\left[\frac{(p-1)(r+1)}{p}\right]^{1 / p}(\alpha-1)^{(p-1-r) / p}\right. \\
& \left.\times\left[\frac{1}{\alpha-1} F\left(\frac{1}{p}, \frac{1}{q+1} ; \frac{q+2}{q+1} ;-\frac{r+1}{q+1}(\alpha-1)^{-(r+1)}\right)+\frac{\Gamma\left(\frac{r+2}{r+1}\right) \Gamma\left(\frac{p-1}{p}\right)}{\Gamma\left(\frac{p r+2 p-r-1}{p r+p}\right)}\right]\right\}
\end{aligned}
$$




$$
\begin{aligned}
= & \lim _{\alpha \rightarrow 1^{+}}\left\{\left[\frac{(p-1)(r+1)}{p}\right]^{1 / p}(\alpha-1)^{(p-1-r) / p}\right. \\
& \left.\times\left[\frac{1}{\alpha-1} F\left(\frac{1}{q+1}, \frac{1}{p} ; \frac{q+2}{q+1} ;-\frac{r+1}{q+1}(\alpha-1)^{-(r+1)}\right)+\frac{\Gamma\left(\frac{r+2}{r+1}\right) \Gamma\left(\frac{p-1}{p}\right)}{\Gamma\left(\frac{p r+2 p-r-1}{p r+p}\right)}\right]\right\} \\
& (\text { by Lemma 3.1(v)) } \\
= & \lim _{\alpha \rightarrow 1^{+}}\left\{[ \frac { ( p - 1 ) ( r + 1 ) } { p } ] ^ { 1 / p } \left[\left(\frac{r+1}{q+1}\right)^{-\frac{1}{q+1}} \frac{\Gamma\left(\frac{q+2}{q+1}\right) \Gamma\left(\frac{q+1-p}{p q+p}\right)}{\Gamma(1) \Gamma\left(\frac{1}{p}\right)}(\alpha-1)^{\frac{(p-1-q)(r+1)}{p q+q}}\right.\right. \\
& \left.\left.+\frac{\Gamma\left(\frac{r+2}{r+1}\right) \Gamma\left(\frac{p-1}{p}\right)}{\Gamma\left(\frac{p r+2 p-r-1}{p r+p}\right)}(\alpha-1)^{(p-1-r) / p}\right]\right\} \text { (by Lemma 3.1(vii)) } \\
= & \infty .
\end{aligned}
$$

(I) (iii) The results $T_{f_{q, r}}(\alpha) \in C((0,1) \cup(1, \infty))$ and $T_{f_{q, r}}(\alpha)$ is strictly increasing on $(0,1)$ follow by Proposition 1.1(i). For $\alpha>1$, by (3.6), we compute that

$$
\begin{aligned}
& T_{f_{q, r}}^{\prime}(\alpha) \\
= & {\left[\frac{(p-1)(r+1)}{p}\right]^{1 / p}\left\{\frac{p-1-r}{p} \frac{\Gamma\left(\frac{r+2}{r+1}\right) \Gamma\left(\frac{p-1}{p}\right)}{\Gamma\left(\frac{p r+2 p-r-1}{p+p}\right)}(\alpha-1)^{-(r+1) / p}\right.} \\
& +\frac{(p-1-q)(r+1)}{p(q+1)} F\left(\frac{1}{p}, \frac{1}{q+1} ; \frac{q+2}{q+1} ;-\frac{r+1}{q+1}(\alpha-1)^{-(r+1)}\right)(\alpha-1)^{-(p+1+r) / p} \\
& \left.-\frac{r+1}{q+1}\left(1+\frac{r+1}{q+1}(\alpha-1)^{-(r+1)}\right)^{-1 / p}(\alpha-1)^{-(p+1+r) / p}\right\} \\
= & {\left[\frac{(p-1)(r+1)}{p}\right]^{1 / p}(\alpha-1)^{-(r+1) / p}\left\{\frac{p-1-r}{p} \int_{0}^{1} \frac{d t}{\left(1-t^{r+1}\right)^{1 / p}}\right.} \\
& +\frac{(p-1-q)(r+1)}{p(q+1)^{2}}(\alpha-1)^{\frac{r-q}{q+1}} \int_{0}^{(\alpha-1)^{-(r+1)}} \frac{x^{\frac{-q}{q+1}}}{\left(1+\frac{r+1}{q+1} x\right)^{1 / p}} d x \\
& \left.-\frac{r+1}{q+1}\left(1+\frac{r+1}{q+1}(\alpha-1)^{-(r+1)}\right)^{-1 / p}(\alpha-1)^{-(p+1+r) / p}\right\}
\end{aligned}
$$

(by Lemma 3.1(iii) and (iv))

$$
\begin{aligned}
= & {\left[\frac{(p-1)(r+1)}{p}\right]^{1 / p}(\alpha-1)^{-(r+1) / p}\left\{\frac{p-1-r}{p} \int_{0}^{1} \frac{d t}{\left(1-t^{r+1}\right)^{1 / p}}\right.} \\
& +\frac{(p-1-q)(r+1)}{p(q+1)}(\alpha-1)^{\frac{r-q}{q+1}} \int_{0}^{(\alpha-1)^{-\frac{r+1}{q+1}}} \frac{d y}{\left(1+\frac{r+1}{q+1} y^{q+1}\right)^{1 / p}} \\
& \left.-\frac{r+1}{q+1}\left(1+\frac{r+1}{q+1}(\alpha-1)^{-(r+1)}\right)^{-1 / p}(\alpha-1)^{-1}\right\}
\end{aligned}
$$

(by setting $x=y^{q+1}$ in the second integral)

$\equiv\left[\frac{(p-1)(r+1)}{p}\right]^{1 / p}(\alpha-1)^{-(r+1) / p} \tilde{H}(\alpha)$ 
where

$$
\begin{aligned}
\tilde{H}(\alpha) \equiv & \frac{p-1-r}{p} \int_{0}^{1} \frac{d t}{\left(1-t^{r+1}\right)^{1 / p}} \\
& +\frac{(p-1-q)(r+1)}{p(q+1)}(\alpha-1)^{\frac{r-q}{q+1}} \int_{0}^{(\alpha-1)^{-\frac{r+1}{q+1}}} \frac{d y}{\left(1+\frac{r+1}{q+1} y^{q+1}\right)^{1 / p}} \\
& -\frac{r+1}{q+1}\left(1+\frac{r+1}{q+1}(\alpha-1)^{-(r+1)}\right)^{-1 / p}(\alpha-1)^{-1}
\end{aligned}
$$

To determine the number of critical points of $T_{f_{q, r}}(\alpha)$ on $(1, \infty)$ is equivalent to determine the number of zeros of $T_{f_{q, r}}^{\prime}(\alpha)$ on $(1, \infty)$; that is, the number of zeros of $\tilde{H}(\alpha)$ on $(1, \infty)$. We compute that

$$
\begin{aligned}
& \tilde{H}^{\prime}(\alpha) \\
&= \frac{(p-1-q)(r+1)}{p(q+1)^{2}}(r-q)(\alpha-1)^{\frac{r-q}{q+1}-1} \int_{0}^{(\alpha-1)^{-\frac{r+1}{q+1}}} \frac{d y}{\left(1+\frac{r+1}{q+1} y^{q+1}\right)^{1 / p}} \\
&-\frac{(p-1-q)(r+1)^{2}}{p(q+1)^{2}}\left(1+\frac{r+1}{q+1}(\alpha-1)^{-(r+1)}\right)^{-1 / p}(\alpha-1)^{-2} \\
&+\frac{r+1}{q+1}\left(1+\frac{r+1}{q+1}(\alpha-1)^{-(r+1)}\right)^{-1 / p}(\alpha-1)^{-2} \\
&-\frac{(r+1)^{3}}{p(q+1)^{2}}\left(1+\frac{r+1}{q+1}(\alpha-1)^{-(r+1)}\right)^{-\frac{p+1}{p}}(\alpha-1)^{-r-3} \text { (by simple calculation) } \\
&= \frac{(p-1-q)(r+1)}{p(q+1)^{2}}(r-q)(\alpha-1)^{\frac{r-q}{q+1}-1} \int_{0}^{(\alpha-1)^{-\frac{r+1}{q+1}}} \frac{d y}{\left(1+\frac{r+1}{q+1} y^{q+1}\right)^{1 / p}} \\
&+\frac{r+1}{q+1}\left(\frac{r+1}{p}-\frac{r-q}{q+1}\right)\left(1+\frac{r+1}{q+1}(\alpha-1)^{-(r+1)}\right)^{-1 / p}(\alpha-1)^{-2} \\
&-\frac{(r+1)^{3}}{p(q+1)^{2}}\left(1+\frac{r+1}{q+1}(\alpha-1)^{-(r+1)}\right)^{-\frac{p+1}{p}}(\alpha-1)^{-r-3} \text { (by simple calculation) } \\
&= \frac{(q-p+1)(r+1)}{p(q+1)^{2}}(q-r)(\alpha-1)^{\frac{r-q}{q+1}-1} \int_{0}^{(\alpha-1)^{-\frac{r+1}{q+1}}} \frac{d y}{\left(1+\frac{r+1}{q+1} y^{q+1}\right)^{1 / p}} \\
&+\left[\frac{(r+1)^{2}}{p(q+1)}\left(1+\frac{r+1}{q+1}(\alpha-1)^{-(r+1)}\right)^{-1 / p}(\alpha-1)^{-2}\right] \\
& \times {\left[\left(1+\frac{p(q-r)}{(q+1)(r+1)}\right)-\frac{q+1}{r+1}(\alpha-1)^{r+1}+1\right.} \\
&> 0 \text { for } \alpha>1 . \\
&(\text { by simple calculation) }
\end{aligned}
$$


So, for $q \geq p-1>r, \tilde{H}(\alpha)$ is strictly increasing on $(1, \infty)$. In addition, we obtain that $\lim _{\alpha \rightarrow 1^{+}} \tilde{H}(\alpha)<0$ and $\lim _{\alpha \rightarrow \infty} \tilde{H}(\alpha)>0$ since $\lim _{\alpha \rightarrow 1^{+}} T_{f_{q, r}}^{\prime}(\alpha)=-\infty$ and $\lim _{\alpha \rightarrow \infty} T_{f_{q, r}}(\alpha)=\infty$. Hence, for fixed $q \geq p-1$, there exists exactly one number $\alpha^{*}=\alpha^{*}(r)>1$ such that $\tilde{H}\left(\alpha^{*}\right)=0$. Therefore, $T_{f_{q, r}}(\alpha)$ has exactly one critical point at $\alpha^{*}$, a local minimum, on $(1, \infty)$.

(II) (i) If $r=p-1$, the results $\lim _{\alpha \rightarrow 0^{+}} T_{f_{q, r}}(\alpha)=0$ and $\lim _{\alpha \rightarrow \infty} T_{f_{q, r}}(\alpha)=$ $(p-1)^{1 / p} \frac{\pi}{p} \csc \frac{\pi}{p}$ follow by Proposition 1.1(ii) and (iv), respectively.

(II) (ii) $\lim _{\alpha \rightarrow 1^{-}} T_{f_{q, r}}(\alpha)=\infty$ by Proposition 1.1(iii). In addition, $\lim _{\alpha \rightarrow 1^{+}} T_{f_{q, r}}$ $(\alpha)=\infty$ by applying (3.14).

(II) (iii) The results $T_{f_{q}}(\alpha) \in C((0,1) \cup(1, \infty))$ and $T_{f_{q}}(\alpha)$ is strictly increasing on $(0,1)$ follow by Proposition 1.1(i). For $\alpha>1, T_{f_{q, r}}^{\prime}(\alpha)<0$ follows easily by (3.15) and (3.16) since $q \geq p-1$. Hence $T_{f_{q, r}}(\alpha)$ is strictly decreasing on $(1, \infty)$.

(III) (i) If $r>p-1$, the results $\lim _{\alpha \rightarrow 0^{+}} T_{f_{q, r}}(\alpha)=0$ and $\lim _{\alpha \rightarrow \infty} T_{f_{q, r}}(\alpha)=0$ follow by Proposition 1.1(ii) and (iv), respectively.

(III) (ii) $\lim _{\alpha \rightarrow 1^{-}} T_{f_{q, r}}(\alpha)=\infty$ by Proposition 1.1(iii). In addition, $\lim _{\alpha \rightarrow 1^{+}}$ $T_{f_{q, r}}(\alpha)=\infty$ by applying (3.14).

(III) (iii) The results $T_{f_{q}}(\alpha) \in C((0,1) \cup(1, \infty))$ and $T_{f_{q}}(\alpha)$ is strictly increasing on $(0,1)$ follow by Proposition 1.1(i). For $\alpha>1, T_{f_{q, r}}^{\prime}(\alpha)<0$ follows easily by (3.15) and (3.16) since $q \geq p-1$. Hence $T_{f_{q, r}}(\alpha)$ is strictly decreasing on $(1, \infty)$.

The proof of Theorem 2.2 is now complete.

Proof of Corollary 2.3. The existence and (exact) multiplicity of positive solutions in Corollary 2.3 follow straight from Theorem 2.2; we omit the details of the proof. Corollary 2.3(A)(I)(vi) is from [4, Corollary 2.2(A)(vi)]. The ordering property of positive solutions obtained in Corollary 2.3 can be proved easily; we omit the proofs. Cf. [4, Proof of Corollary 2.2].

\section{The Proof of Lemma 3.1}

For Lemma 3.1(i)-(iii), (v)-(ix), (xi), and (xv), see [10, pp. 46-47, 54, 56, 368-369, 371]. For Lemma 3.1(iv), see [2, p. 333, formula 3.194.1]. For Lemma 3.1(xiii), see [1, p. 62, formula 3.2.8]. Lemma 3.1(x), (xii) and (xiv) can be proved easily by (2.1) and (2.3); we omit them. Finally, the proofs of Lemma 3.1(xvi) and (xvii) are easy but tedious; we omit them.

\section{ACKNOWLEDGMENTS}

Much of the computation in this paper has been checked using computer algebra package Mathematica 7.0. 


\section{REFERENCES}

1. G. Gasper and M. Rahman, Basic hypergeometric series, Cambridge University Press, Cambridge, 1990.

2. I. S. Gradshtein, I. M. Ryzhik and A. Jeffrey, Table of integrals, series, and products, Academic Press, Boston, 1994.

3. T. Laetsch, The number of solutions of a nonlinear two point boundary value problem, Indiana Univ. Math. J., 20 (1970), 1-13.

4. S.-Y. Lee, J.-Y. Liu, S.-H. Wang and C.-P. Ye, A complete classification of bifurcation diagrams of a $p$-Laplacian Dirichlet problem, J. Math. Anal. Appl., 330 (2007), 276-290.

5. S.-Y. Lee, S.-H. Wang and C.-P. Ye, Explicit necessary and sufficient conditions for the existence of a dead core solution of a $p$-Laplacian steady-state reaction-diffusion problem, Discrete Contin. Dyn. Syst. 2005, suppl., pp. 587-596.

6. P. L. Lions, On the existence of positive solutions of semilinear elliptic equations, SIAM Review, 24 (1982), 441-467.

7. H. O. Peitgen, D. Saupe and K. Schmitt, Nonlinear elliptic boundary value problems versus their finite difference approximations: numerically irrelevant solutions, J. Reine Angew. Math., 322 (1981), 74-117.

8. J. B. Seaborn, Hypergeometric functions and their applications, Texts in Applied Mathematics, Vol. 8, Springer-Verlag, New York, 1991.

9. J. Smoller and A. Wasserman, Global bifurcation of steady-state solutions, J. Differential Equations, 39 (1981), 269-290.

10. S. Zhang and J. Jin, Computation of special functions, John Wiley \& Sons, New York, 1996.

Feng-Lin Wang and Shin-Hwa Wang

Department of Mathematics

National Tsing Hua University

Hsinchu 300, Taiwan

E-mail: g933252@oz.nthu.edu.tw shwang@math.nthu.edu.tw 